\title{
Article
}

\section{Alien Pulse Rejection in Concurrent Firing LIDAR}

\author{
Gunzung Kim ${ }^{1} \oplus$, Jeongsook Eom ${ }^{2}$ and Yongwan Park ${ }^{3, *}$ \\ 1 Institute of Information and Communication, Gyeongsan 38541, Gyeongbuk, Korea; gzkim@yu.ac.kr \\ 2 Department of Multimedia and Communication Engineering, Yeungnam University, Gyeongsan 38541, \\ Gyeongbuk, Korea; jseom@yu.ac.kr \\ 3 Department of Information and Communication Engineering, Yeungnam University, Gyeongsan 38541, \\ Gyeongbuk, Korea \\ * Correspondence: ywpark@yu.ac.kr
}

Citation: Kim, G.; Eom, J.; Park, Y. Alien Pulse Rejection in Concurrent Firing LIDAR. Remote Sens. 2022, 14, 1129. https://doi.org/10.3390/ rs14051129

Academic Editor: Andrzej Stateczny

Received: 10 January 2022

Accepted: 23 February 2022

Published: 24 February 2022

Publisher's Note: MDPI stays neutral with regard to jurisdictional claims in published maps and institutional affiliations.

Copyright: (c) 2022 by the authors. Licensee MDPI, Basel, Switzerland. This article is an open access article distributed under the terms and conditions of the Creative Commons Attribution (CC BY) license (https:/ / creativecommons.org/licenses/by/ $4.0 /)$.

\begin{abstract}
Mobile pulse light detection and ranging (LIDAR) is an essential component of autonomous vehicles. The obstacle detection function of autonomous vehicles requires very low failure rates. With an increasing number of autonomous vehicles equipped with LIDAR sensors for use in the detection and avoidance of obstacles and for safe navigation through the environment, the probability of mutual interference becomes an important issue. The reception of foreign laser pulses can lead to problems such as ghost targets or a reduced signal-to-noise ratio (SNR). In this paper, we presented the probability that any LIDAR sensor would interfere mutually by considering spatial and temporal overlaps. We presented some typical mutual interference scenarios in real-world vehicle applications, as well as an analysis of the interference mechanism. We proposed a new multi-plane LIDAR sensor which used coded pulse streams encoded by carrier-hopping prime code (CHPC) technology to measure surrounding perimeters without mutual interference. These encoded pulses utilized a random azimuth identification and checksum with random spreading code. We modeled the entirety of the LIDAR sensor operation in Synopsys OptSim and represented the alien pulse elimination functionality obtained via modeling and simulation.
\end{abstract}

Keywords: LIDAR sensor; direct time-of-flight; mutual interference; alien pulse; concurrent firing; carrier-hopping prime code; autonomous vehicle

\section{Introduction}

Range sensors are devices that capture the three-dimensional (3-D) structure of the world from the viewpoint of the sensor, usually measuring distances to closest targets [1-5]. These measurements could be across a scanning plane or a 3-D image with distance measurements at every point.

These range sensors have been used for many years in the fields of localization, obstacle detection and tracking for autonomous vehicles [6,7]. These can be employed as measurement devices to address the joint problem of online tracking and the detection of the current modality [8]. These can be helpful indicators for use in sustainability assessment by presenting the distribution characteristics of each sustainability index for vehicles [9]. Obstacle detection functions of autonomous vehicles require low failure rates [10]. Interference is inherent to all active sensors and wireless applications with the same or an overlapping frequency range [11-14]. In general, interference describes the coherent superposition of two or more different waves at one point in space. A wave is a rotating vector in the complex plane, where the addition of several vectors results in deterministic changes to magnitude and phase. Signal amplitude can be decreased (destructive interference) or increased (constructive interference). Single interference can be treated as one-shot noise or error and can be eliminated using the Kalman filter or the particle learning technique $[15,16]$.

Within a few years, the adaption rate of vehicular radio detection and ranging (radar) systems will have drastically increased in this newly emerging market [17]. In terms of 
safety-related applications, interference risk will particularly threaten further proliferation if harmful mutual interference occurs [18-20]. It is too late to find efficient and pragmatic countermeasures to avoid apparent interference risk when severe interference problems cause malfunction or out-of-order situations in safety radar devices [14,21]. The only reasonable and valid approach is to counteract these issues before the problems are manifested. The EU funding project, more safety for all by radar interference mitigation (MOSARIM), started in January 2010, intending to investigate possible automotive radar interference mechanisms via both simulation and real-world road tests $[18-20,22]$. Interference from an identical pulsed radar sensor with the same pulse repetition frequency (PRF) generates a ghost target at a constant distance. If the radars are similar, and only have slightly different PRFs, then a ghost target will appear as a large target moving slowly in the range. The apparent velocity is dependent on the difference between the two PRFs and could be anything from a couple of millimeters per second up to a couple of hundred meters per second. A slightly different PRF results in a moving ghost target, as the time difference between the transmitted pulse and the interfering pulse increases or decreases from pulse repetition period to pulse repetition period. If the PRFs are vastly different, then more than one ghost target could appear per cycle [11,23]. Appropriate countermeasures and mitigation techniques were studied and assessed, and general guidelines and recommendations were developed [11,23-25]. The ideas for countermeasures were extracted and structured into six basic categories. From the six basic categories, a list of 22 variants was compiled, evaluated, and ranked concerning various criteria for the implementation effort, required power and computational resources, cost, the requirement for harmonization, etc. They selected the top nine countermeasures for further evaluation in order to derive guidelines. Their mitigation performance ranged from a few $\mathrm{dB}$ up to (theoretically) infinite $\mathrm{dB}$. Their accurate and consistent application to all new automotive radar products will result in close to interference-free automotive radar operations.

Time-of-flight (ToF) range cameras acquire a 3-D image of a scene simultaneously for all pixels from a single viewing location. Optical interference between a sensor and another sensor is a crucial matter [26]. It is not easy to separate the amplitude-modulated wave from the sensor itself and another sensor once they are mixed. This shows how the mixed pixel and multipath separation algorithm can be applied to range imaging measurements projected into Cartesian coordinates. Significant improvements in accuracy can be achieved in particular situations where the influence of multipath interference frequently causes distortions [27-29]. The multiple return separation algorithm requires a consistent and known relationship between the cameras' amplitudes and phase responses at both modulation frequencies. This type of calibration was not available for these cameras, so it was carried out by comparing manually selected regions of the image judged to be minimally affected by multipath interference. If these regions did contain multipath, the calibration would be compromised, leading to poor performance. Another source of potential error is distance linearity measurement inaccuracies, which are common in uncalibrated range cameras. It is also possible that the optimization algorithm encountered local minima, producing an incorrect answer.

The light detection and ranging (LIDAR) sensor is one of the essential perception sensors for autonomous vehicles that can travel autonomously [1-7,10]. A LIDAR sensor installed on an autonomous vehicle lets this vehicle generate a detailed 3-D local map of its environment. Histogram analysis of the 3-D local map is used to select the obstacle candidates. It incorporates matched candidates into results from previous scans and updates the positions of all obstacles based on vehicle motion before the following scan. The car then takes these maps and combines them with high-resolution global maps of the world, producing different types of maps that allow it to drive itself. The reception of foreign laser pulses can lead to ghost targets or a reduced signal-to-noise ratio. The reception of unwanted laser pulses from other LIDAR sensors is called mutual interference between LIDAR sensors [30-37]. Concretely, interference effects in automotive LIDAR sensors are caused by the superposition of disturbances from other LIDAR sensors at the 
receiving antenna with the incoming coherent use signals being reflected from objects within the LIDAR sensor's detection zone. The simplest method for the prevention of such interference is to install each sensor by tilting them forward $2^{\circ}$ to $3^{\circ}$. However, that is not enough. Another measure is to emit as few lasers as possible. The laser should be off where sensor data are unnecessary. According to the analyzed result, the laser was automatically off in unnecessary areas and scans were curtailed. These functions prevent interference and extend laser devices and reduce power consumption. Moreover, one more step is ready for use, which is a function where motor speed is slightly changed on command. A difference in motor speed that is more than 300 almost prevents interference [38].

Until now, interference has not been considered as a problem because the number of vehicles equipped with LIDAR sensors was small, and, as a result, the possibility of interference was extremely unlikely. So, despite a predicted higher number of LIDAR sensors, the possibility of interference-induced problems has to be reduced considerably. With the increase in the number of autonomous vehicles equipped with LIDAR sensors for use in obstacle detection and avoidance for safe navigation through environments, the possibility of mutual interference becomes an issue. Imagine massive traffic jams occurring in the morning and evening. What if all of these vehicles were equipped with LIDAR sensors? With a growing number of autonomous vehicles equipped with LIDAR sensors operating close to each other at the same time, LIDAR sensors may receive laser pulses from other LIDAR sensors.

This article is structured as follows: in Section 2, the occurrence of mutual interference in radar and LIDAR sensors is discussed. The causes of mutual interference and the extensive study of radar sensor interference in the MOSARIM project are briefly described. In Section 3, a new multi-plane LIDAR sensor, which uses coded pulse streams to measure surrounding perimeters without mutual interference, is presented. In Section 4, three relevant mutual interference scenarios in real-world vehicle applications of simulations are presented. In Section 5, four different simulation phases are set up according to the operating mode of two LIDAR sensors, and their results are shown. In each scenario, LIDAR sensors operated in single-pulse mode suffered from mutual interference, but LIDAR sensors with a coded pulse steam mode effectively rejected alien pulses. In Section 6, the conclusion is given, and future research paths are suggested. uls

\section{Occurrence of Mutual Interference}

Some studies have been performed regarding the depiction of the occurrence of mutual interference between LIDAR sensors and improvement of the design of pulsed LIDAR sensors. Kim et al. [30-33] presented two LMS-511 LIDAR sensors that operated simultaneously in a closed space, resulting in mutual interference. Popko et al. [35,36] carried out similar experiments with two LIDAR sensors. In their articles, signal intersections occurring between sensors were analyzed, and based on this, a geometric proximity model for mutual interference was presented. They introduced the terms direct interference and indirect interference. Direct interference occurs when two LIDAR sensors are oriented at each other, and a signal from one is coupled into the other's receiver. Indirect interference, or scattering interference, occurs when the target scattering of one LIDAR sensor's signal is received by another. Unlike radar sensors, where direct interference accounts for the majority of cases of interference, indirect interference makes up almost the majority of interferences generated by LIDAR sensors. Hwang et al. [37] investigated the characteristics and impacts of mutual interference with regard to a LIDAR sensor using an analog true-random signal for autonomous vehicle applications. Lo et al. [39] proposed a proof-of -principle LIDAR and demonstrated the superiority of two-dimensional (2-D) LIDAR modulation on interference robustness and the elimination of the near-far effect.

\subsection{Direct Time-of-Flight and Mutual Interference}

A LIDAR sensor measures the distance to an object on the direct time-of-flight (dToF) principle based on the known speed of light $\left(299,792,458 \mathrm{~m} \mathrm{~s}^{-1}\right)$ by emitting an object with 
a laser pulse and analyzing the reflected laser pulse. As illustrated in Figure 1, it transmits a $5 \mathrm{~ns}$ to $20 \mathrm{~ns}$ laser pulse toward an object and measures the time taken by the laser pulse to be reflected off the object and returned to the sender. The incoming laser pulse causes a delay depending on the distance of the object. The LIDAR sensor calculates that a distance to a target is half the round-trip transit time multiplied by the speed of light.
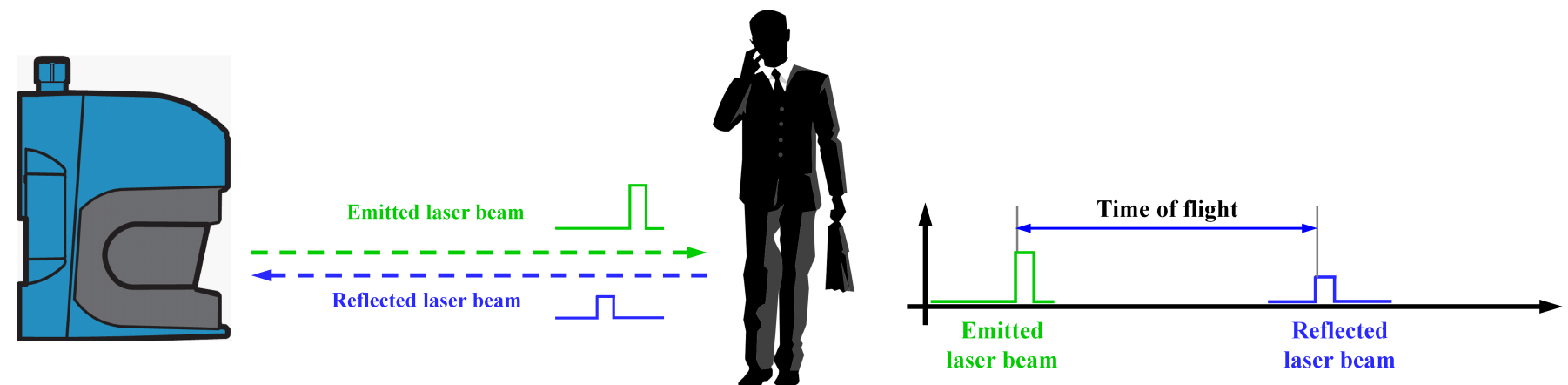

(a)

(b)

Figure 1. Normal direct time-of-flight in a LIDAR sensor. A LIDAR sensor measures distance by actively emitting an object with a laser pulse and a receiver that is sensitive to the laser's wavelength to capture the reflected laser pulse. The sensor measures the time-of-flight $\Delta t$ between when the laser pulse is emitted and when the reflected laser pulse is received by the sensor. The time-of-flight is proportional to twice the distance between the sensor and the object (round-trip); therefore, the distance can be estimated as distance $=c \frac{\Delta t}{2}$, where $c$ is the speed of light. (a) Normal direct time-of-flight situation; (b) normal direct time-of-flight measurement.

Furthermore, LIDAR sensors are exposed to various emissions from other users, such as LIDAR sensors in other cars at near distances and other LIDAR sensors roadside or on closed roads. As shown in Figure 2, multiple LIDAR sensors operate in proximity to each other and thus generate mutual interference. If two or more LIDAR sensors that are in proximity to each other transmit pulses in the same operating frequency band, each LIDAR sensor system will receive reflected signals of the other LIDAR sensors. These reflected signals are more or less indistinguishable from reflections from their targets. These received interference signals can be of similar amplitude and can confuse receiving systems and target displays. This well-known problem manifests itself in increases in false alarm rates and in undesirable losses of sensitivity for the detection of targets. Mutual interference is a severe problem for many types of LIDAR sensors and leads to a continuing problem for future LIDAR sensor concepts. 


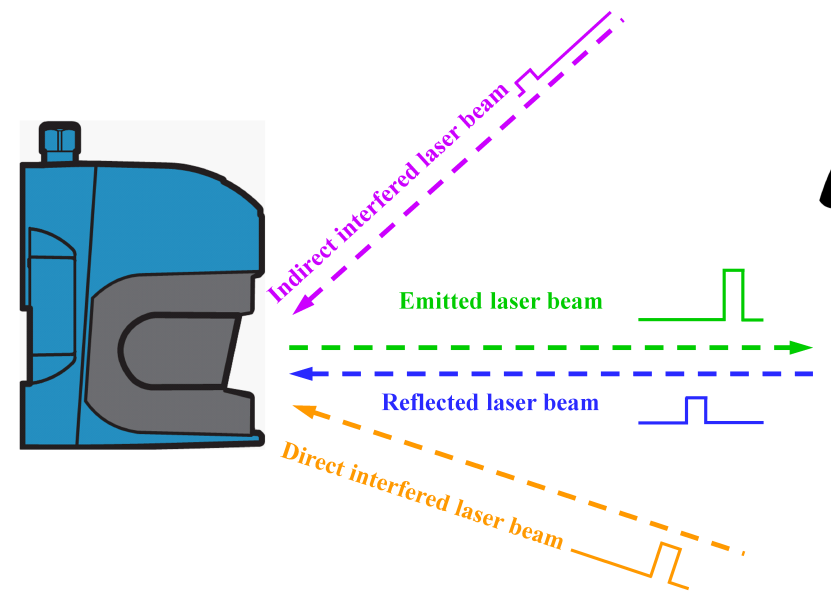

(a)
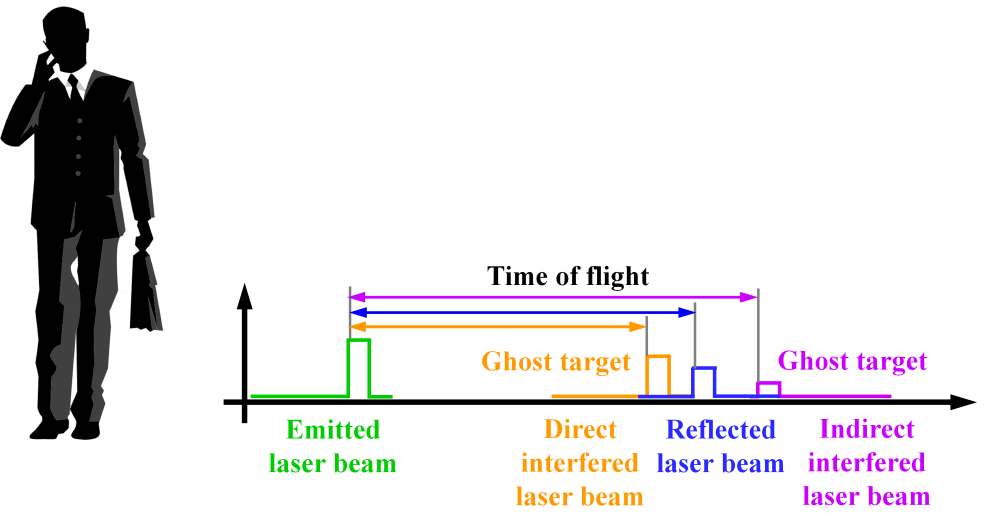

(b)

Figure 2. Interfered direct time-of-flight in the LIDAR sensor. This LIDAR sensor and other LIDAR sensors emitted a laser pulse to measure the distance of an object. This LIDAR sensor received several reflected laser pulses within a specific measuring time. The first received laser pulse is a direct laser pulse emitted from another LIDAR sensor. The second received laser pulse is its own reflected laser pulse. The third received laser pulse is a reflected laser pulse emitted from another LIDAR sensor. All these received laser waveforms have similar pulse shapes with different amplitudes. Therefore, the LIDAR sensor cannot distinguish which laser pulse is its own laser pulse. (a) Interfered direct time-of-flight situation; (b) interfered direct time-of-flight measurement.

\subsection{Relevant Mutual Interference Scenarios in Real-World Vehicle Applications}

In order to investigate radar sensor interference in the MOSARIM project, they selected scenarios that were particularly relevant to be considered for analysis in simulation and practical measurements based on the partner's experience [19]. The scenario selection took into account a number of different factors, apart from obvious direct interference from one radar sensor to another. According to relevant vehicle applications in which radar sensors are typically used to detect surrounding vehicles or objects, they divide these mutual interference scenarios into two main categories: direct interference scenarios as listed in Tables 1-4 and indirect interference scenarios as listed in Tables 5-7.

Table 1. Relevant direct interference traffic scenarios of forward-looking radar sensors without further target objects.

\begin{tabular}{lll}
\hline Scenario Name & Main Scenario Factor & Potential Interference Effect \\
\hline Standstill, short and long distance & $\begin{array}{l}\text { Stationary victim vehicle and interferer } \\
\text { in direct field of view }\end{array}$ & Occurrence of ghost targets \\
\hline Victim approaches interferer & $\begin{array}{l}\text { Victim approaches stationary interferer } \\
\text { in direct field of view }\end{array}$ & $\begin{array}{l}\text { Occurrence of ghost targets, observation of } \\
\text { signal/noise ratio (SNR) while approaching }\end{array}$ \\
\hline Victim and interferer pass each other & $\begin{array}{l}\text { Radar sensor interferes within each } \\
\text { other vehicle's radar main-lobe }\end{array}$ & Occurrence of ghost target \\
\hline
\end{tabular}

Table 2. Relevant direct interference traffic scenarios of forward-looking radar sensors with rear- or sideward-looking sensors.

\begin{tabular}{lll}
\hline Scenario Name & Main Scenario Factor & Potential Interference Effect \\
\hline $\begin{array}{l}\text { Traffic in same direction with similar velocities, } \\
\text { rear sensor }\end{array}$ & $\begin{array}{l}\text { Victim drives on the same lane behind } \\
\text { the interfering vehicle }\end{array}$ & $\begin{array}{l}\text { Interference-increased noise may make } \\
\text { interfering vehicle invisible }\end{array}$ \\
\hline
\end{tabular}


Table 2. Cont.

\begin{tabular}{lll}
\hline Scenario Name & Main Scenario Factor & Potential Interference Effect \\
\hline Overtaking, forward sensor & $\begin{array}{l}\text { Victim overtakes the interferer, victim } \\
\text { looks forward, and interferer looks to } \\
\text { the side or rear }\end{array}$ & Detection disturbed by interfering radar \\
\hline Interference with multiple rear-looking sensors & Multiple interferers & Detection disturbed by interfering radar \\
\hline
\end{tabular}

Table 3. Relevant direct interference traffic scenarios of forward-looking radar sensors with presence of further target objects.

\begin{tabular}{|c|c|c|}
\hline Scenario Name & Main Scenario Factor & Potential Interference Effect \\
\hline Standstill at intersection, target ahead & $\begin{array}{l}\text { Stationary host vehicle and interferer } \\
\text { with presence of neutral vehicle }\end{array}$ & Loss of target neutral vehicle \\
\hline Victim approaches target at intersection & $\begin{array}{l}\text { Victim approaches stationary interferer } \\
\text { with presence of neutral vehicle }\end{array}$ & Loss of target neutral vehicle \\
\hline Following a target, oncoming interferer & $\begin{array}{l}\text { Interfering vehicle approaches and faces } \\
\text { host vehicle }\end{array}$ & $\begin{array}{l}\text { Loss of target neutral vehicle, degradation of } \\
\text { target parameter estimation }\end{array}$ \\
\hline Oncoming interferer in road curve & Road curve & $\begin{array}{l}\text { Host vehicle realizes false tracking because } \\
\text { interferer vehicle approaches. Degradation of } \\
\text { the target parameter estimation }\end{array}$ \\
\hline $\begin{array}{l}\text { Oncoming interferer with motorcycle } \\
\text { target }\end{array}$ & Weak target motorcycle in long range & $\begin{array}{l}\text { Tracking and/or detection of motorcycle } \\
\text { disturbed by vehicle driving in opposite } \\
\text { direction. Degradation of SNR leading to no } \\
\text { detection of motorcycle }\end{array}$ \\
\hline $\begin{array}{l}\text { Oncoming interferer with pedestrian as } \\
\text { target }\end{array}$ & Weak target pedestrian in close range & $\begin{array}{l}\text { Victim vehicle radar disturbed by approaching } \\
\text { interferer. Victim vehicle radar detects } \\
\text { interferer, but does not detect pedestrian }\end{array}$ \\
\hline $\begin{array}{l}\text { Victim and interferer drive in parallel, } \\
\text { target ahead }\end{array}$ & $\begin{array}{l}\text { Interferer drives in same direction as } \\
\text { victim vehicle }\end{array}$ & $\begin{array}{l}\text { Loss of target neutral vehicle. Degradation of } \\
\text { target parameter estimation }\end{array}$ \\
\hline $\begin{array}{l}\text { Victim and interferer drive in parallel, } \\
\text { target ahead, lane change }\end{array}$ & Target neutral vehicle lane change & Degradation of target parameter estimation \\
\hline Interference from crossing traffic & Weak target pedestrian in close range & Degradation of SNR leading to no detection \\
\hline $\begin{array}{l}\text { High density of interferers at } \\
\text { intersection }\end{array}$ & High density of interferers & $\begin{array}{l}\text { Host vehicle is static and disturbed by many } \\
\text { interfering radar sensors. No detection, blind } \\
\text { in some areas, sustained false target }\end{array}$ \\
\hline Roundabout & $\begin{array}{l}\text { Victim and interferer do not face each } \\
\text { other directly }\end{array}$ & $\begin{array}{l}\text { False negative, lost of target neutral vehicle, no } \\
\text { collision warning }\end{array}$ \\
\hline
\end{tabular}

Table 4. Relevant direct interference traffic scenarios of rear- and sideward-looking radar sensors.

\begin{tabular}{llll}
\hline Scenario Name & Main Scenario Factor & Potential Interference Effect \\
\hline $\begin{array}{l}\text { Direct interference, oncoming traffic, } \\
\text { both sensors face the rear }\end{array}$ & $\begin{array}{l}\text { High density of sensors, direct dazzling, } \\
\text { potential guard rail, jersey barrier, or } \\
\text { tunnel }\end{array}$ & Reduced detection performance \\
\hline $\begin{array}{l}\text { Direct interference, oncoming traffic, } \\
\text { sensors look to side }\end{array}$ & High density of sensors, direct dazzling & Reduced detection performance \\
\hline $\begin{array}{l}\text { Direct interference, traffic in the same } \\
\text { direction with similar velocities, side } \\
\text { sensor }\end{array}$ & $\begin{array}{l}\text { Interferer on next lane with similar } \\
\text { velocity to the victim, sensors look to } \\
\text { the side }\end{array}$ & $\begin{array}{l}\text { Interference-increased noise may hinder } \\
\text { detection of vehicle }\end{array}$ & \\
\hline
\end{tabular}


Table 4. Cont.

\begin{tabular}{|c|c|c|c|}
\hline Scenario Name & Main Scenario Factor & Potential Interference Effect & \\
\hline Overtaking & $\begin{array}{l}\text { Interfering vehicle overtakes the victim } \\
\text { vehicle, sensors look to the side }\end{array}$ & $\begin{array}{l}\text { Interference-increased noise } \\
\text { detection of vehicle }\end{array}$ & hinder \\
\hline Being overtaken & $\begin{array}{l}\text { Interfering vehicle overtaken by the } \\
\text { victim vehicle, sensor looks to the side }\end{array}$ & $\begin{array}{l}\text { Interference-increased noise } \\
\text { detection of vehicle }\end{array}$ & hinder \\
\hline Overtaking, rear sensing & $\begin{array}{l}\text { Victim vehicle overtakes the interfering } \\
\text { vehicle, victim sensor faces the rear, } \\
\text { interfering sensor looks to the side }\end{array}$ & $\begin{array}{l}\text { Interference-increased noise } \\
\text { detection of vehicle }\end{array}$ & hinder \\
\hline Being overtaken, side sensing & $\begin{array}{l}\text { Interference vehicle overtakes the victim } \\
\text { vehicle, interferer sensor faces the rear, } \\
\text { victim sensor looks to the side }\end{array}$ & $\begin{array}{l}\text { Interference-increased noise } \\
\text { detection of vehicle }\end{array}$ & hinder \\
\hline $\begin{array}{l}\text { Forward-looking sensor on following } \\
\text { vehicle }\end{array}$ & $\begin{array}{l}\text { Interferer drives on the same lane behind } \\
\text { the victim vehicle }\end{array}$ & $\begin{array}{l}\text { Interference-increased noise } \\
\text { interfering vehicle invisible }\end{array}$ & may make \\
\hline $\begin{array}{l}\text { Road approaches with various angles } \\
\text { other than } 90^{\circ}\end{array}$ & $\begin{array}{l}\text { Two roads approach with various angles, } \\
\text { sensors look to the side and/or interferer } \\
\text { looks forward }\end{array}$ & Reduced detection performance & \\
\hline
\end{tabular}

Table 5. Relevant indirect interference traffic scenarios of forward-looking radar sensors.

\begin{tabular}{lll}
\hline Scenario Name & Main Scenario Factor & Potential Interference Effect \\
\hline $\begin{array}{l}\text { Indirect interference with rear-looking } \\
\text { sensor (truck) }\end{array}$ & Interferer and truck as neutral vehicle & Detection disturbed by interfering radar \\
\hline $\begin{array}{l}\text { Indirect interference with rear-looking } \\
\text { sensor (guard rail) }\end{array}$ & Guard rail, jersey barrier, and tunnel & Detection disturbed by interfering radar \\
\hline $\begin{array}{l}\text { Indirect interference with multiple } \\
\text { rear-looking sensors }\end{array}$ & $\begin{array}{l}\text { Guard rail, jersey barrier, tunnel, and multiple } \\
\text { interferer sources }\end{array}$ & Detection disturbed by interfering radar \\
\hline $\begin{array}{l}\text { Indirect interference with passing } \\
\text { vehicles and presence of guard rail }\end{array}$ & $\begin{array}{l}\text { Two vehicle approaching each other. Guard rail } \\
\text { or jersey barrier }\end{array}$ & $\begin{array}{l}\text { Non-sustained false target. Detection } \\
\text { degradation }\end{array}$ \\
\hline $\begin{array}{l}\text { Indirect interference with following } \\
\text { vehicle and presence of guard rail }\end{array}$ & $\begin{array}{l}\text { Interferer follows victim within guard rail or } \\
\text { jersey barrier }\end{array}$ & $\begin{array}{l}\text { Non-sustained false target. Detection } \\
\text { degradation }\end{array}$ \\
\hline
\end{tabular}

Table 6. Relevant indirect interference traffic scenarios of rear- and sideward-looking radar sensors with presence of further target objects.

\begin{tabular}{lll}
\hline Scenario Name & Main Scenario Factor & Potential Interference Effect \\
\hline Victim and interferer drive in parallel, target follows & $\begin{array}{l}\text { Target approaches victim, victim } \\
\text { is disturbed by interferer }\end{array}$ & Late detection of neutral vehicle \\
\hline Blind spot detection with multiple interferers & Multiple interference sources & Loss of target neutral vehicle \\
\hline Interference with forward-looking radar & High density of sensors & $\begin{array}{l}\text { Interferer driving on the same lane } \\
\text { behind the victim vehicle, possible loss } \\
\text { of target }\end{array}$ \\
\hline Being overtaken, side sensing & $\begin{array}{l}\text { Interfering vehicle overtakes the } \\
\text { victim vehicle, interferer sensor } \\
\text { looks forward, victim sensor } \\
\text { looks to the side }\end{array}$ & $\begin{array}{l}\text { Late detection of interferer } \\
\end{array}$ \\
\hline
\end{tabular}


Table 7. Relevant indirect interference traffic scenarios of rear- and sideward-looking radar sensors.

\begin{tabular}{lll}
\hline Scenario Name & Main Scenario Factor & Potential Interference Effect \\
\hline Guard rail & $\begin{array}{l}\text { Victim and interferer drive parallel along guard rail } \\
\text { or jersey barrier }\end{array}$ & $\begin{array}{l}\text { Radar signal is reflected by the guard } \\
\text { rail or jersey barrier }\end{array}$ \\
\hline $\begin{array}{ll}\text { Intersection, sensors face the rear } \\
\text { Intersection, victim looks to the } \\
\text { side, interferer sensor faces the rear }\end{array}$ & $\begin{array}{l}\text { Intersection, victim looks to the side, interferer } \\
\text { sensor faces the rear }\end{array}$ & Reduced detection performance \\
$\begin{array}{l}\text { Intersection, interferer looks to the } \\
\text { side, victim sensor faces the rear }\end{array}$ & $\begin{array}{l}\text { Intersection, interferer looks to the side, victim } \\
\text { sensor faces the rear }\end{array}$ & Reduced detection performance \\
\hline $\begin{array}{l}\text { Intersection, interfering vehicle } \\
\text { turns, interferer sensors look to the } \\
\text { side, victim sensor faces the rear }\end{array}$ & $\begin{array}{l}\text { Intersection, interfering vehicle turns, interfering } \\
\text { sensors look to the side, victim sensor faces car }\end{array}$ & Reduced detection performance \\
\hline $\begin{array}{l}\text { Intersection, victim vehicle turns, } \\
\text { victim sensors look to the side, } \\
\text { interferer sensor faces the rear }\end{array}$ & $\begin{array}{l}\text { Intersection, victim vehicle turns, victim sensors look } \\
\text { to the side, interferer sensor faces car }\end{array}$ & Reduced detection performance \\
\hline $\begin{array}{l}\text { Intersection, interference with } \\
\text { sideward-looking radar }\end{array}$ & High density of sensors & Reduced detection performance \\
\hline $\begin{array}{l}\text { Parking slot, interferer sensor looks } \\
\text { forward }\end{array}$ & High density of sensors, direct dazzling & $\begin{array}{l}\text { Interference-increased noise may hinder } \\
\text { detection of interferer }\end{array}$ \\
\hline $\begin{array}{l}\text { Parking slot, interference with } \\
\text { sideward-looking sensor }\end{array}$ & Parking slot, interferer sensor looks to the side & $\begin{array}{l}\text { Interference-increased noise may hinder } \\
\text { detection of interferer }\end{array}$ \\
\hline
\end{tabular}

\section{Signal Processing of Concurrent Firing LIDAR Sensor without Mutual Interference}

In this section, we proposed a new multi-plane LIDAR sensor, which uses coded pulse streams encoded by carrier-hopping prime code (CHPC) technology to measure surrounding perimeters without mutual interference. These encoded pulses utilized a random azimuth identification and checksum with random spreading code. We modeled the entirety of the LIDAR sensor operation in Synopsys OptSim with Mathworks MATLAB and represented the alien pulse elimination functionality obtained via modeling and simulation. We chose parameters based on our previous prototype LIDAR sensor [40] for optical characteristics related to laser transmission and reception, pulse reflection, lens, etc. Three scenarios of simulation and their results are shown, according to the arrangement of two LIDAR sensors. In each scenario, two LIDAR sensors were operated in single-pulse mode and/or coded pulse steam mode.

In the previous paper [41], we proposed a LIDAR sensor that changes the measurement strategy from a sequential to a concurrent firing and measuring method. The proposed LIDAR utilized a 3-D scanning LIDAR method that consisted of 128 output channels in one vertical line in the measurement direction and concurrently measured the distance for each of these 128 channels. The LIDAR sensor emitted 128 coded pulse streams encoded by CHPC technology with identification and checksum. The emission channel could be recognized when the reflected pulse stream was received and demodulated. This information could estimate the time when the laser pulse stream was emitted and calculate the distance to the object reflecting the laser. By identifying the received reflected wave, even if several positions were measured simultaneously, the measurement position could be recognized after the reception. In this paper, we describe a more detailed description of the alien pulse rejection method of the CHPC encoder and decoder as presented in Figure 3.

In order to generate a coded pulse stream to be transmitted, three pieces of information are required. A coded pulse stream to be transmitted can only be generated when there is an azimuth identification (ID) indicating the currently measured angle, a cyclic redundancy check (CRC) that checks whether there is an error when receiving data, and a spreading code for the generation of a coded pulse stream. The proposed LIDAR sensor generates azimuth ID and spreading code randomly, preventing collision with other LIDAR sensors 
using the same method or inference from the outside. The three pieces of information are stored in the internal memory and decode the reflected signal. When a reflected waves are received, these are converted into a pulse stream, decoded using the spreading code stored in the internal memory, and then the demodulation process is performed. Only when the pulse stream has the same spreading code in the internal memory is it deemed suitable and proceeds to the next step. If a different spreading code is used, it is judged inappropriate and then discarded. Data determined to be suitable are checked for data errors through the CRC inspection process. Data without errors move to the next step. If the error-free data have the same azimuth ID used for encoding, the distance to the destination is calculated using the difference between the time the azimuth ID was transmitted and the time it was received. Otherwise, the distance is discarded. It is only processed if all three pieces of information match the information transmitted in receiving reflected waves and calculating the distance. Otherwise, it is discarded to remove all information that causes mutual interference from the outside, fundamentally blocking the occurrence of mutual interference.

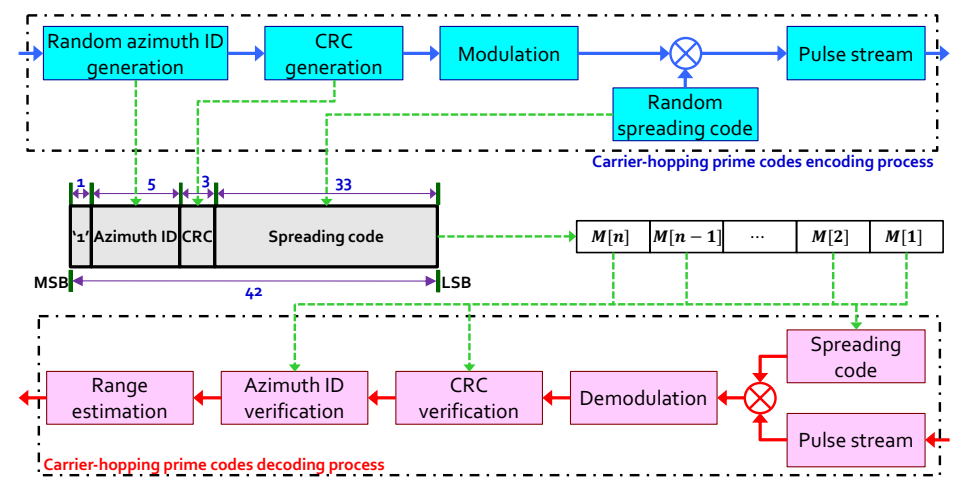

Figure 3. Signal processing aspect of concurrent firing LIDAR sensor.

\section{Simulation Scenarios in Real-World Vehicle Applications}

We selected three relevant scenarios that can occur in indirect interference for analysis in practical measurements. The selection of scenarios took into account several different factors: indirect interference by LIDAR sensor reflection on other objects, the geometry of LIDAR sensors, and static objects such as jersey barriers. In this paper, we focus on running simulations for three scenarios. The first scenario is for indirect interference with two vehicles approaching each other and a jersey barrier (Figure 4a). Indirect interference with a following vehicle and a jersey barrier is the second (Figure $4 \mathrm{~b}$ ). The last is two vehicles driving parallel along a jersey barrier (Figure 4c).

In each scenario, two LIDAR sensors were positioned inside the scene. These two LIDAR sensors operated in two operating modes according to the simulation scenarios. One mode was the coded pulse stream mode based on the concurrent firinging LIDAR sensor, and the other mode was the single-pulse mode based on Velodyne's VLS-128. Two operating modes of LIDAR sensor are summarized in Table 8. These LIDAR sensors were laser measurement sensors that scanned the surrounding perimeter on 128 planes. The light source was a near-infrared laser with a wavelength of $1550 \mathrm{~nm}$. It corresponded to laser class 1 (eye-safe) as per EN 60825-1 (2007-3). It measured in the spherical coordinate system in which each point was determined by the distance from a fixed point and an angle from a fixed direction. It emitted $1550 \mathrm{~nm}$ pulsed laser pulses using laser diodes. If the laser pulse was reflected from the target object, the distance to the object was calculated by the time required for the pulsed laser pulse to be reflected and received by the sensor. For the simple simulation, azimuthal scanning took place in the sector of $170^{\circ}$. To measure the distance and reflected intensity of the surrounding perimeter in the scenarios, the Synopsys OptSim optical simulation software was used for optical characteristics related to laser transmission/reception, and the MathWorks software MATLAB was used for encoding and decoding, signal processing, intensity calculation, and distance calculation tasks [40-42]. 


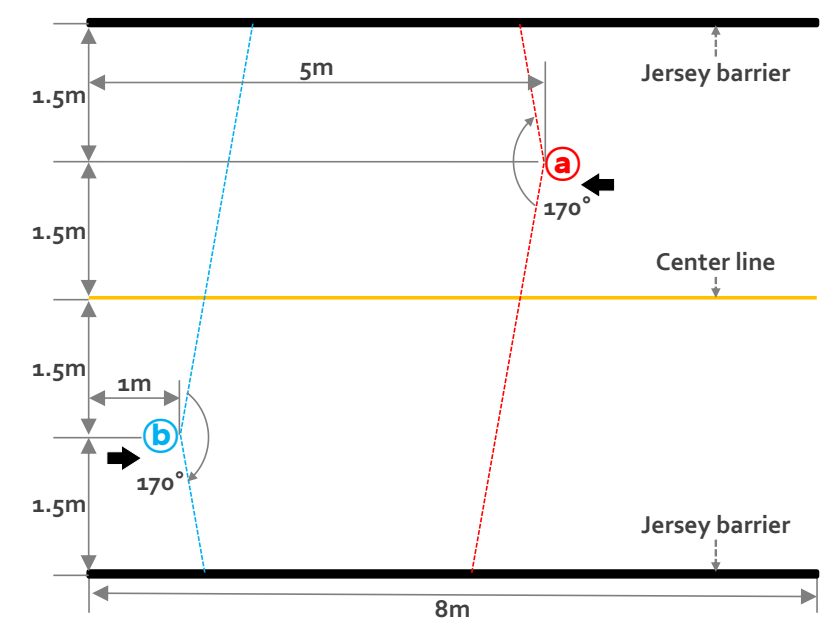

(a)

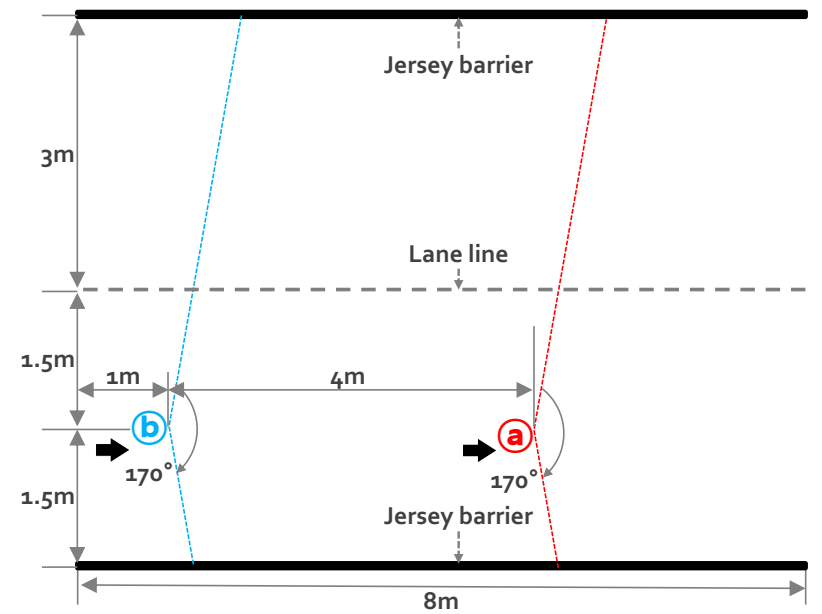

(b)

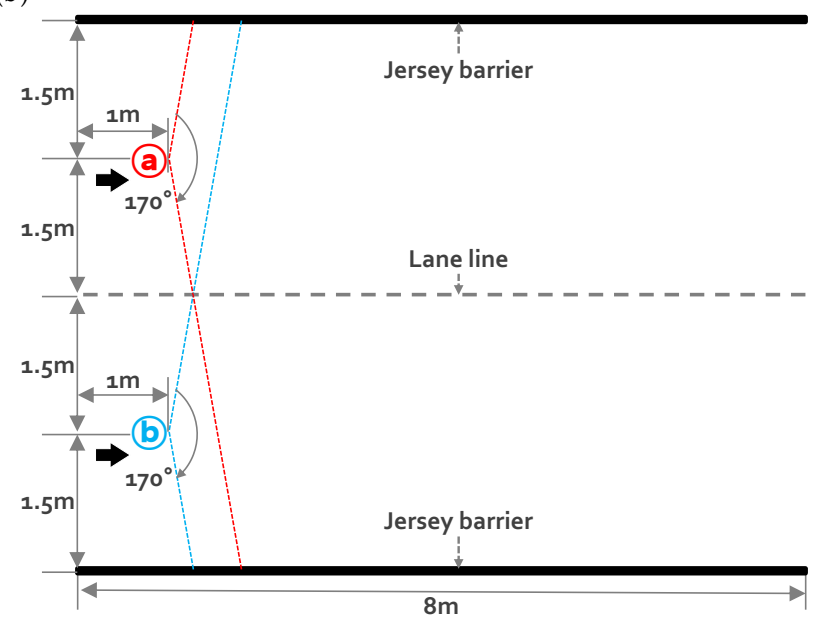

(c)

Figure 4. Simulation scenarios. Black thick lines are jersey barriers, black dotted lines are lane lines, black arrows show moving direction of vehicle, blue and red dotted lines are boundaries of scanning area. (a) Scene \#1: one vehicle with LIDAR sensor (a) and another vehicle with LIDAR sensor (b) are approaching each other and going along a jersey barrier; (b) scene \#2: one vehicle with LIDAR sensor (b) is following vehicle ahead with LIDAR sensor (a) and going along a jersey barrier; and (c) scene \#3: one vehicle with LIDAR sensor (a) and another vehicle with LIDAR sensor (b) are driving parallel along a jersey barrier. 
Table 8. Comparison of two operating modes.

\begin{tabular}{|c|c|c|c|}
\hline \multicolumn{2}{|c|}{ Operating Mode } & Coded Pulse Stream Mode & Single-Pulse Mode \\
\hline \multicolumn{2}{|c|}{ Reference model } & Concurrent firing LIDAR sensor & Velodyne VLS-128 \\
\hline \multicolumn{2}{|c|}{ Scene resolution } & $20,000 \times 128 @ 25 F P S$ & $3600 \times 128 @ 5 F P S$ \\
\hline \multirow{2}{*}{ Field of view (FoV) } & Azimuth & $360^{\circ}\left(-180^{\circ}\right.$ to $\left.180^{\circ}\right)$ & $360^{\circ}\left(-180^{\circ}\right.$ to $\left.180^{\circ}\right)$ \\
\hline & Elevation & $40^{\circ}\left(-20^{\circ}\right.$ to $\left.20^{\circ}\right)$ & $40^{\circ}\left(-25^{\circ}\right.$ to $\left.15^{\circ}\right)$ \\
\hline \multirow{2}{*}{ Angular resolution } & Azimuth & $0.018^{\circ}$ & $0.1^{\circ}$ \\
\hline & Elevation & $0.315^{\circ}$ & $0.11^{\circ}$ (minimum) \\
\hline \multirow{2}{*}{ Bit stream } & Length & 9 bit & $\mathrm{N} / \mathrm{A}$ \\
\hline & Structure & 1 bit Start-of-frame, 5 bit azimuth ID, and 3 bit CRC & $\mathrm{N} / \mathrm{A}$ \\
\hline \multirow{2}{*}{ Spreading method } & Code & Carrier-hopping prime code & $\mathrm{N} / \mathrm{A}$ \\
\hline & Factor & Weight 3 , length 11 & $\mathrm{~N} / \mathrm{A}$ \\
\hline \multicolumn{2}{|c|}{ Number of chips } & 99 per wavelength, 297 per channel & $\mathrm{N} / \mathrm{A}$ \\
\hline \multicolumn{2}{|c|}{ Number of laser pulses } & 27 & 1 \\
\hline \multicolumn{2}{|c|}{ Laser wavelength } & $1530.0413 \mathrm{~nm}$ to $1568.3623 \mathrm{~nm}$ (12.5 GHz spacing) & $1550 \mathrm{~nm}$ \\
\hline \multicolumn{2}{|c|}{ Pulse frequency } & $200 \mathrm{MHz}$ & $\mathrm{N} / \mathrm{A}$ \\
\hline \multicolumn{2}{|c|}{ Pulse width } & $5 \mathrm{~ns}$ & $5 \mathrm{~ns}$ \\
\hline \multicolumn{2}{|c|}{ Firing time } & $1485 \mathrm{~ns}$ & $5 \mathrm{~ns}$ \\
\hline \multicolumn{2}{|c|}{ Firing method } & All channels at once, one chip by one chip & 8 fires at once, 16 firing groups \\
\hline \multicolumn{2}{|c|}{ Time per angle } & $2 \mu \mathrm{s}$ & $53.3 \mu \mathrm{s}$ \\
\hline
\end{tabular}

The scenarios consisted of four steps to identify the occurrence and elimination of mutual interference, and for each step, two LIDAR sensors operated in a predetermined mode. The training phase was carried out in each scenario first, and the mutual interference testing phases were performed later. LIDAR sensors ran and recorded measured data continuously. The analysis of recorded data shows the occurrence and influence of mutual interference.

The first step was the training phase, and normal LIDAR sensor operation was demarcated in the simulation scenarios. To specify the normal range of the distance measured by the two LIDAR sensors, this step consisted of two independent substeps. In the first substep, LIDAR sensor (a) was the normal operating state of the single-pulse mode, but LIDAR sensor (b) was an aborted state. In the second substep, they worked the opposite way. In these two substeps, we recorded the measured distance data at the normal operating LIDAR sensor for four working hours. We calculated the average distance, the maximum distance, and the minimum distance at each angle. After that, we calculated the upper and lower tolerance, as shown in Equations (1)-(5). The LIDAR sensor had two errors; one was a systematic error, and the other was a random statistical error. According to the rule of thumb, adding a slight margin to the maximum and minimum values could reduce misjudgment of the normal distance as interference.

$$
\begin{aligned}
& \bar{d}=\frac{1}{n} \sum_{i=1}^{i=n} d_{i} \\
& d_{\text {max }}=\operatorname{MAX}\left[d_{1}, d_{2}, \ldots, d_{n}\right] \\
& d_{\text {min }}=\operatorname{MIN}\left[d_{1}, d_{2}, \ldots, d_{n}\right] \\
& d_{\text {upper }}=d_{\text {max }}+\left(d_{\text {max }}-\bar{d}\right) \times 0.1 \\
& d_{\text {lower }}=d_{\text {min }}+\left(d_{\text {min }}-\bar{d}\right) \times 0.1
\end{aligned}
$$

The second step to the fourth step was a testing phase. It examined abnormal LIDAR sensor operation in the simulation scenarios. Two LIDAR sensors were in the normal operating state and ran simultaneously. We recorded the measured distance data from the both LIDAR sensor (a) and (b) for 24 hours. We analyzed the recorded data and classified out-of-tolerance distance as the interfered measurement result, as shown in Equation (6). 
In the second step, both LIDAR sensor (a) and (b) were operated in the single-pulse mode. In the third step, LIDAR sensor (a) was the coded pulse stream mode, but LIDAR sensor (b) was in the single-pulse mode. In the fourth step, two LIDAR sensor (a) and (b) were operated in the coded pulse stream mode.

$$
f(d)= \begin{cases}\text { normal distance } & \text { if } d_{\text {lower }} \leq d \leq d_{\text {upper }} \\ \text { interfered distance } & \text { else. }\end{cases}
$$

\section{Simulation Results}

\subsection{First Step: Training Phase for Making Demarcated LIDAR Sensor Operation}

Figures 5-7 are the results of the training phase, the first step in the simulation. They show the 2-D distance grid map acquired by LIDAR sensor (a) and (b) in each scenario. As described in Section 4, two LIDAR sensors were operated in the single-pulse mode separately. Green color-filled square symbols indicate correct distance data. Scatter plots are composed of many superimposed green color-filled square symbols. These plots show the position of the jersey barrier and another LIDAR sensor that the LIDAR sensor detects correctly, and they are exactly matched with the simulation scenarios. We calculated the upper and lower tolerance in each scenario, as shown in Equations (1)-(5).

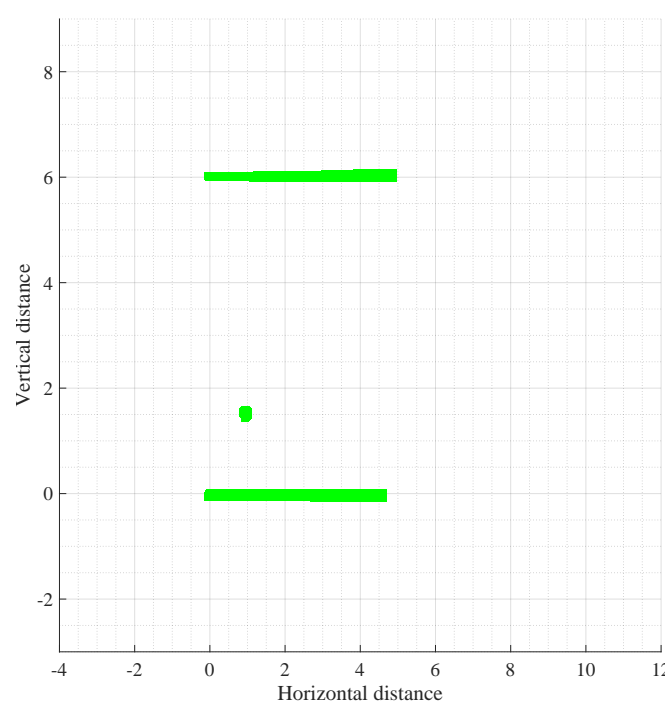

(a)

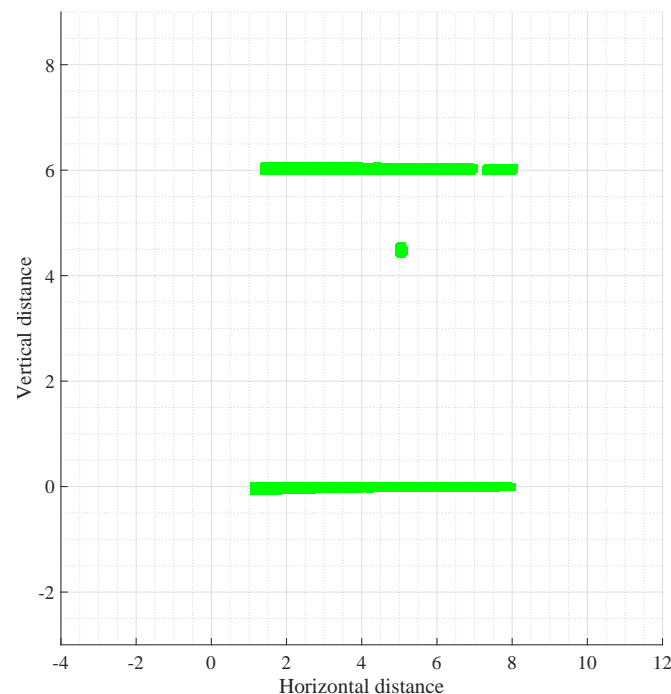

(b)

Figure 5. Distance grid maps of scene \#1: two vehicles are approaching each other and going along a jersey barrier. (a) From LIDAR sensor (a)'s point of view at near top-right corner; (b) LIDAR sensor (b)'s point of view at near bottom-left corner. 


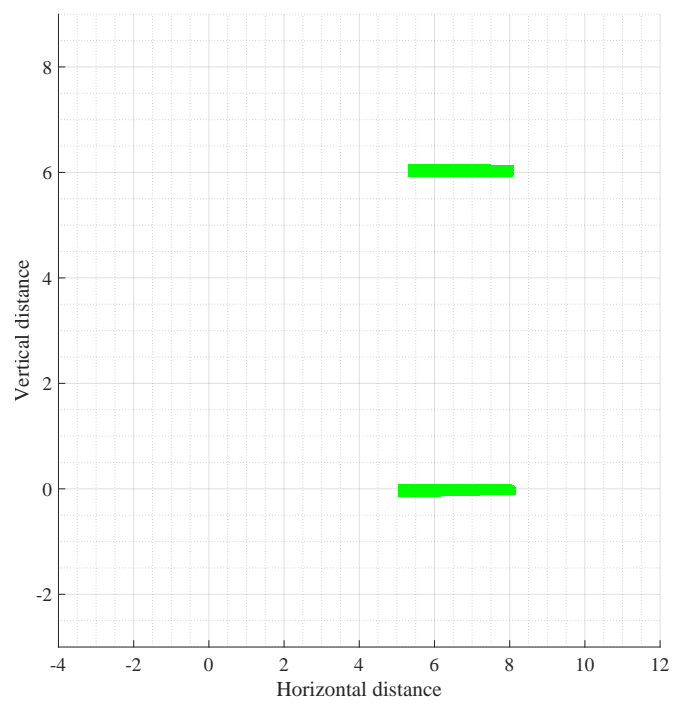

(a)

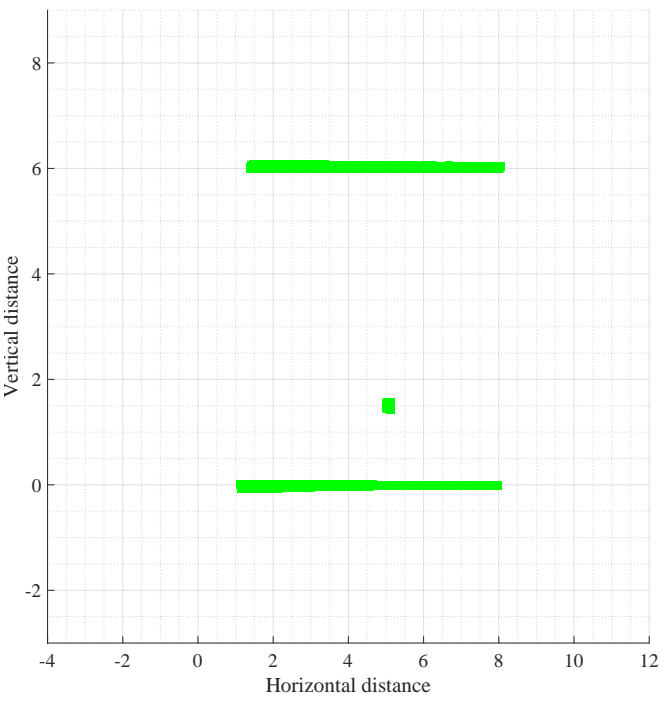

(b)

Figure 6. Distance grid maps of scene \#2: one vehicle is following vehicle ahead and going along a jersey barrier. (a) From LIDAR sensor (a)'s point of view at near bottom-right corner; (b) LIDAR sensor (b)'s point of view at near bottom-left corner.

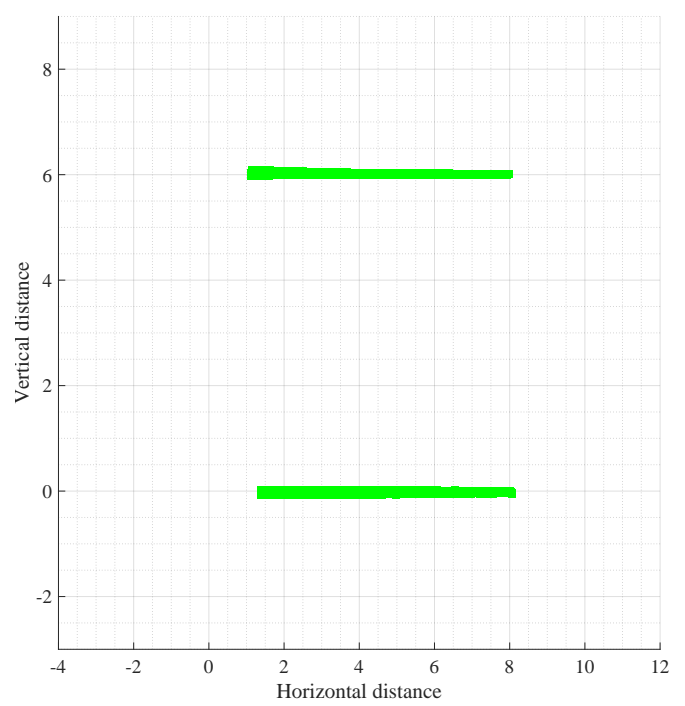

(a)

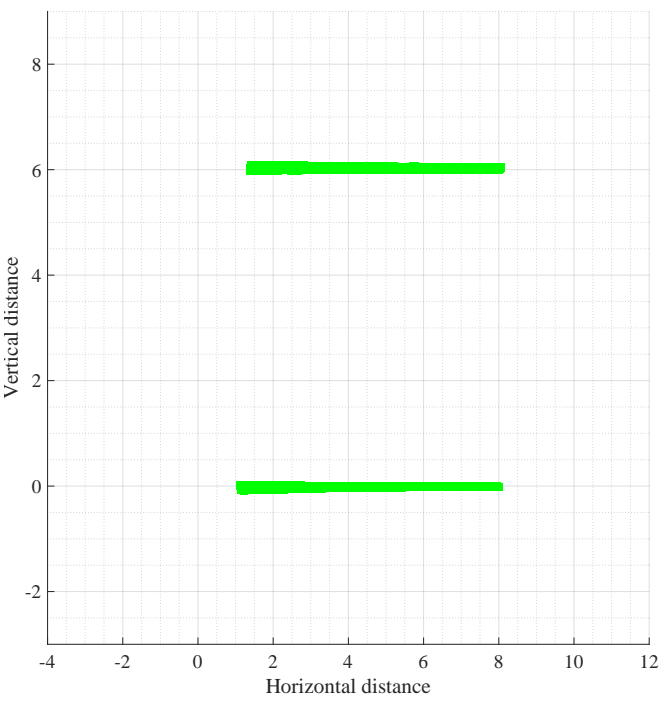

(b)

Figure 7. Distance grid maps of the scene \#3: one vehicle with LIDAR sensor (a) and another vehicle with LIDAR sensor (b) are driving parallel along a jersey barrier. (a) From LIDAR sensor (a)'s point of view at near top-left corner; (b) LIDAR sensor (b)'s point of view at near bottom-left corner.

\subsection{Second Step: Occurrence of Mutual Interference between Single-Pulse Mode}

Figures 8-10 are the results of the second step in the simulation. As described in Section 4, two LIDAR sensors were operated in the single-pulse mode simultaneously. After simulation, we analyzed the recorded data and determined each measured distance to be a correct distance or out-of-tolerance incorrect distance, as shown in Equation (6). Green color-filled square symbols indicate correct distance data, and red cross symbols indicate incorrect distance data. Correct positions indicate LIDAR sensors carry out detections correctly. However, wrong positions indicate that one LIDAR sensor is affected by another LIDAR sensor, which is called a ghost target. The scanning LIDAR sensor uses a spherical coordinate system to represent an object's distance. The closer to the LIDAR sensor, the higher the density of the azimuthal angle and the higher the distance data density.

The six figures show that both LIDAR sensors operated simultaneously in single-pulse mode, and incorrect distance due to mutual interference was measured in both LIDAR 
sensors (a) and (b). As the single-pulse mode calculated the distance using all of the received reflected waves, the laser pulse transmitted by itself was reflected and received. Furthermore, the laser pulse transmitted by another LIDAR sensor, reflected, and received was used for distance calculation. Due to this, when other LIDAR sensors operated within a sufficient effective distance, mutual interference occurred, as shown in these figures.

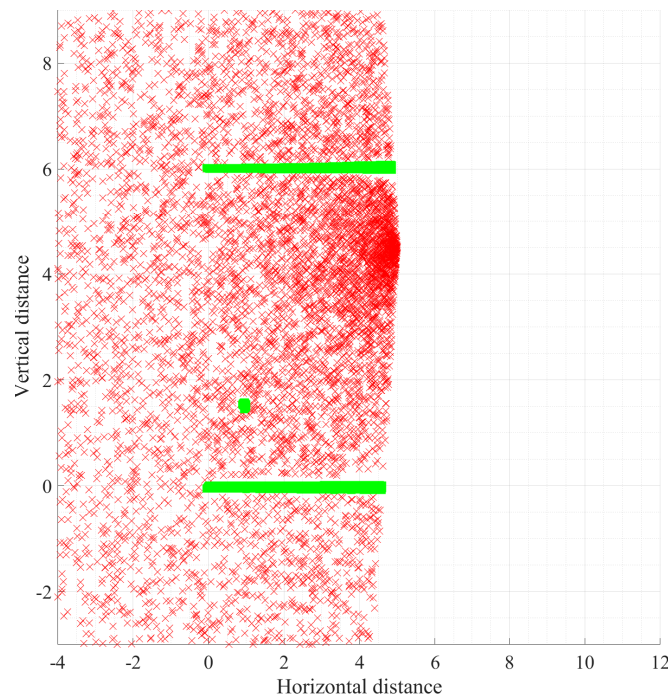

(a)

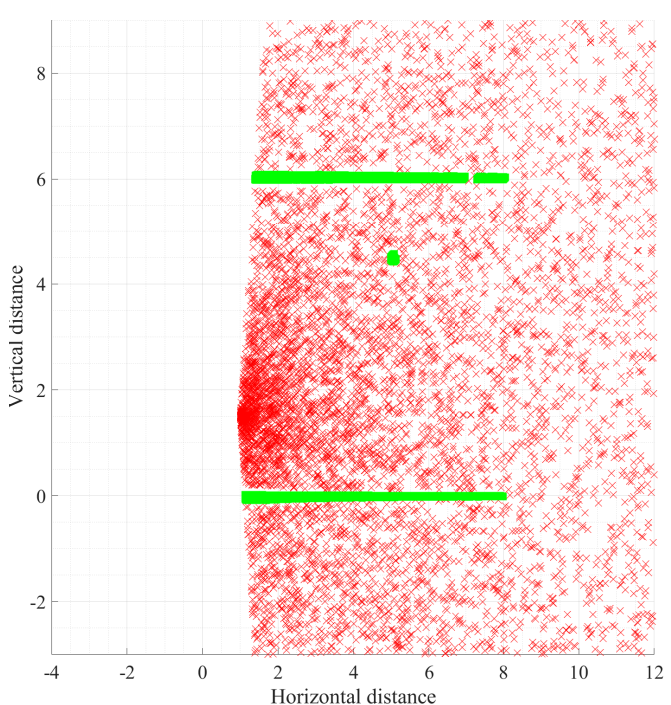

(b)

Figure 8. Distance grid maps of scene \#1 show correct distances and incorrect distances measured by each LIDAR sensor in the second step. (a) From LIDAR sensor (a)'s point of view; (b) LIDAR sensor (b)'s point of view.

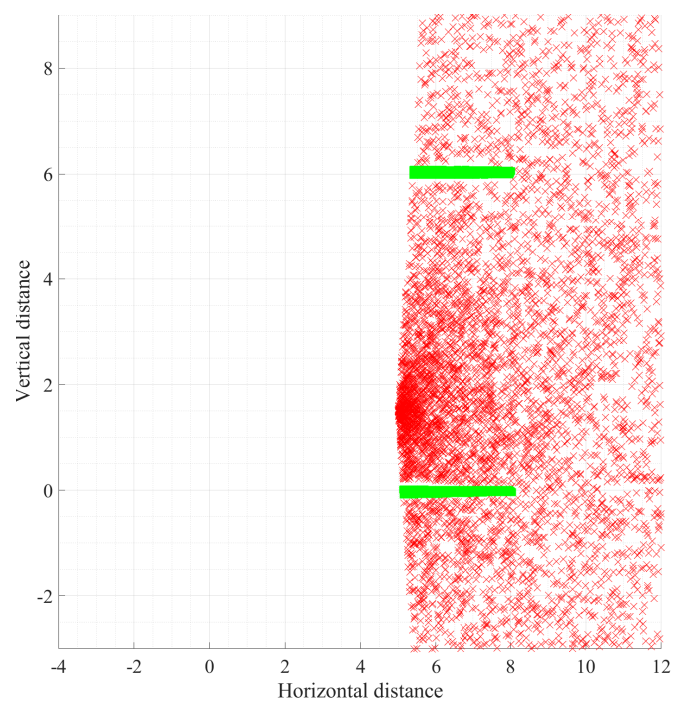

(a)

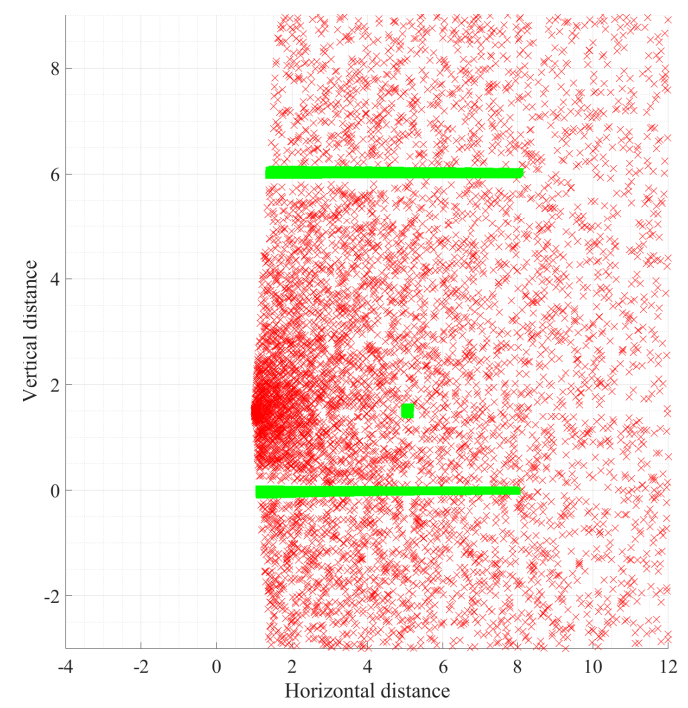

(b)

Figure 9. Distance grid maps of scene \#2 show correct distances and incorrect distances measured by each LIDAR sensor in the second step. (a) From LIDAR sensor @'s point of view; (b) LIDAR sensor (b)'s point of view. 


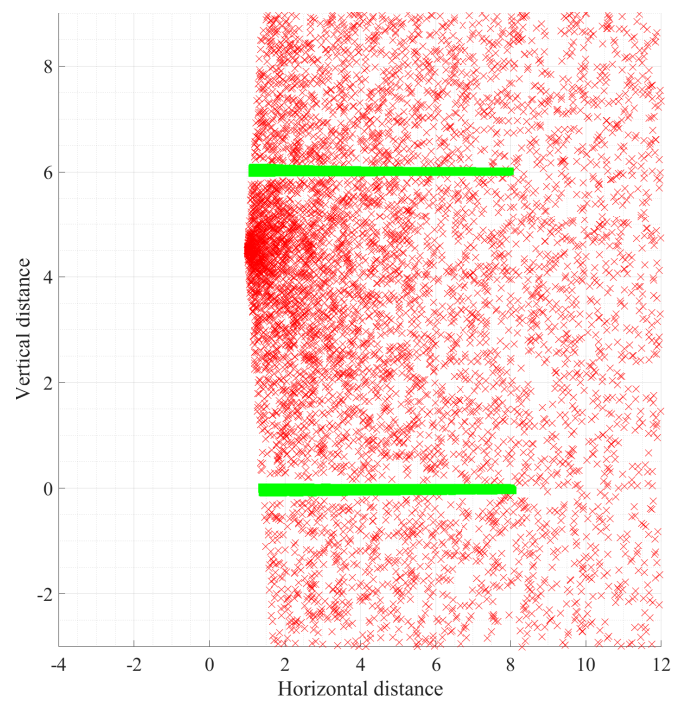

(a)

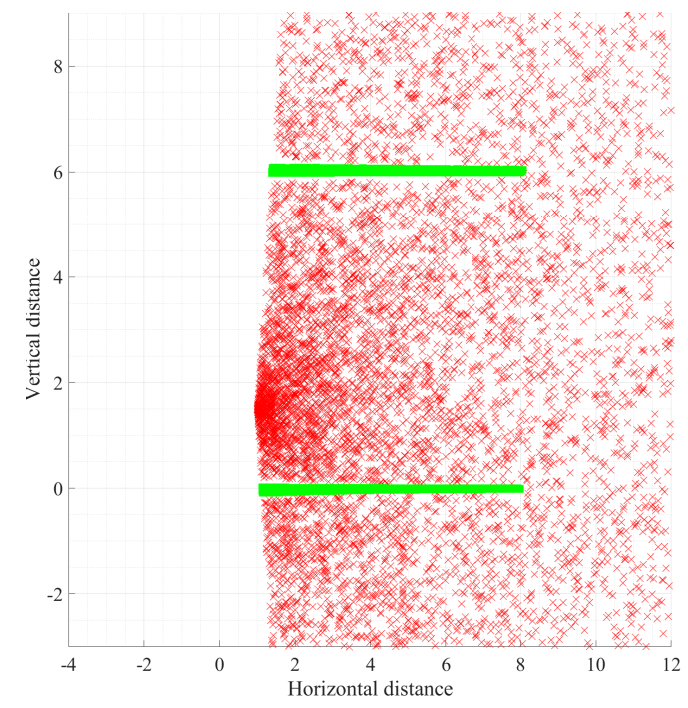

(b)

Figure 10. Distance grid maps of scene \#3 show correct distances and incorrect distances measured by each LIDAR sensor in the second step.(a) From LIDAR sensor @'s point of view; (b) LIDAR sensor (b)'s point of view.

\subsection{Third Step: Occurrence of Mutual Interference in Single-Pulse Mode and Elimination in} Coded Pulse Stream Mode

Figures 11-13 are the results of the third step in the simulation. They show the 2-D distance grid map acquired by LIDAR sensor (a) and (b) in each scenario. As described in Section 4, two LIDAR sensors were operated simultaneously, but LIDAR sensor (a) was operated in the coded pulse stream mode and LIDAR sensor (b) was operated in the single-pulse mode. After simulation, we analyzed and determined the recorded data, as shown in Equation (6), and plotted correct distances as green color-filled square symbols and incorrect distances as red cross symbols.

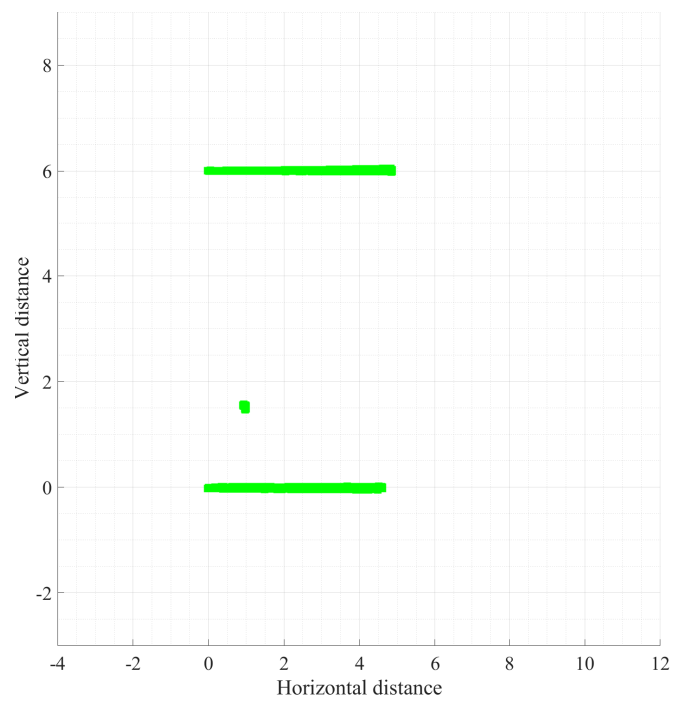

(a)

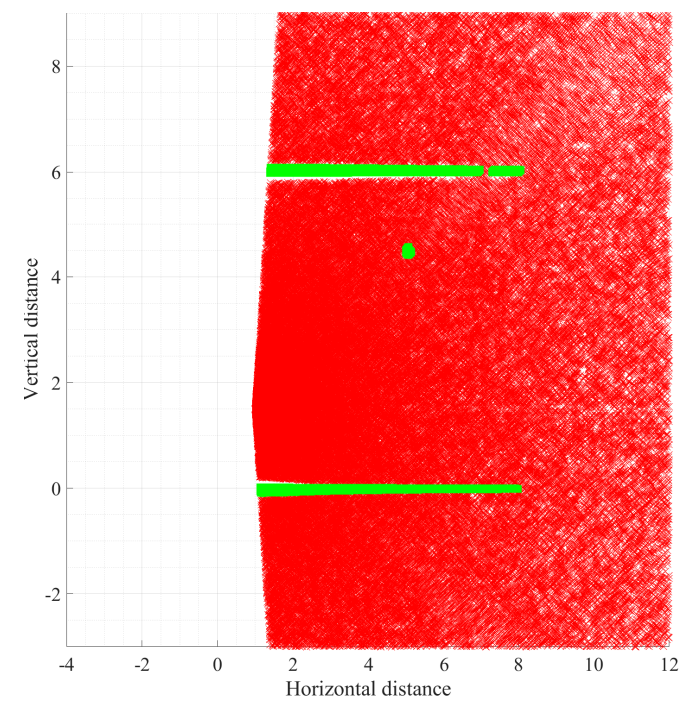

(b)

Figure 11. Distance grid maps of scene \#1 show correct distances and incorrect distances measured by each LIDAR sensor in the third step. (a) From LIDAR sensor (a)'s point of view; (b) LIDAR sensor (b)'s point of view. 


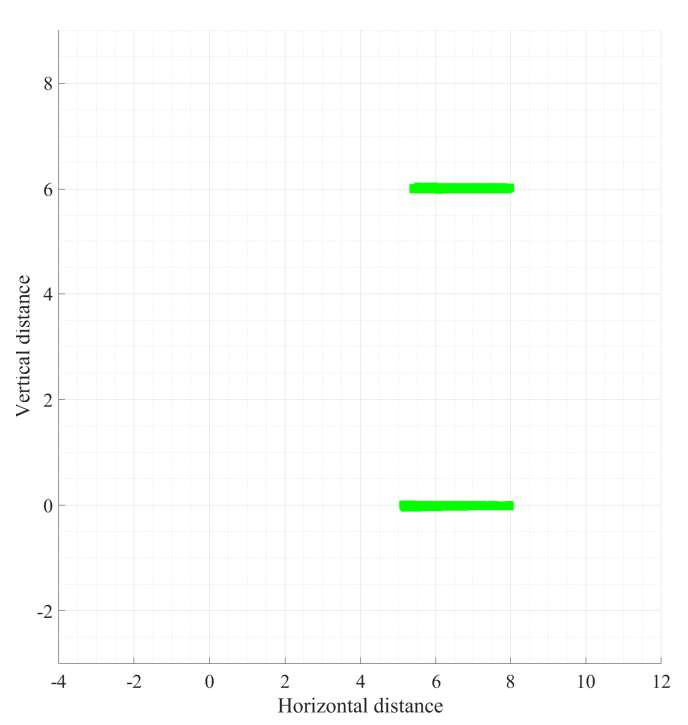

(a)

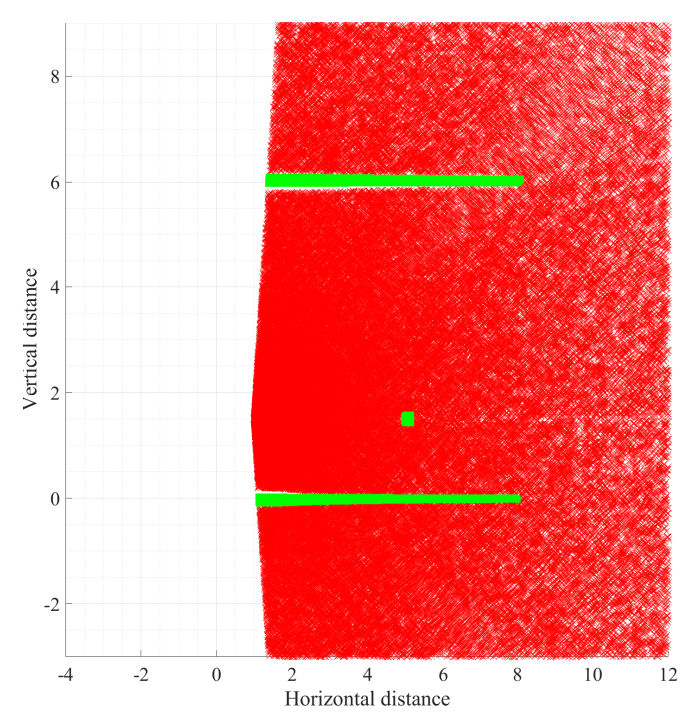

(b)

Figure 12. Distance grid maps of scene \#2 show correct distances and incorrect distances measured by each LIDAR sensor in the third step.(a) From LIDAR sensor @'s point of view; (b) the LIDAR sensor (b)'s point of view.

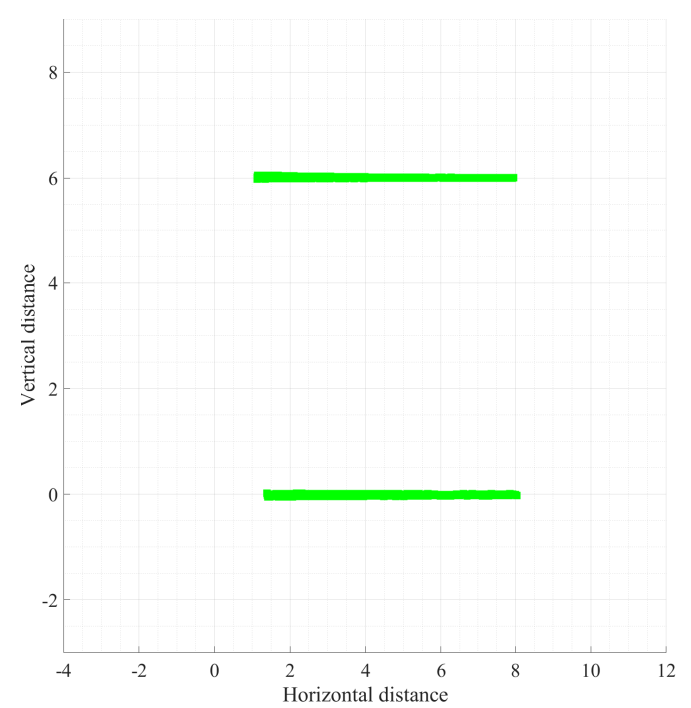

(a)

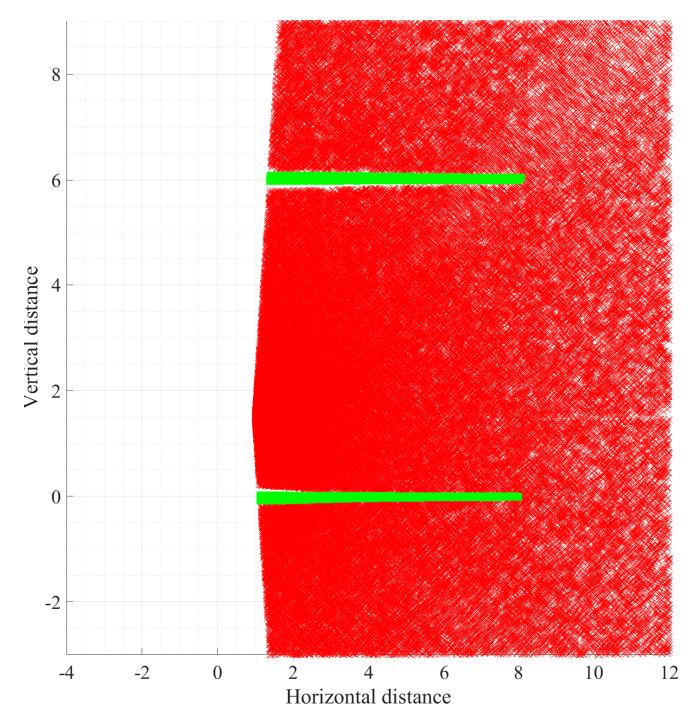

(b)

Figure 13. Distance grid maps of scene \#3 show correct distances and incorrect distances measured by each LIDAR sensor in the third step. (a) From LIDAR sensor (a)'s point of view; (b) LIDAR sensor (b)'s point of view.

In the second step, the two LIDAR sensors operated in single-pulse mode. However, in the third step, only LIDAR sensor (b) operated in single-pulse mode, and LIDAR sensor (a) operated in coded pulse stream mode. The coded pulse stream mode operated differently from the single-pulse mode in two aspects. Multiple pulses were used in the coded pulse stream mode to measure the distance to one place. Even if multiple pulses were received using the code generated by a specific rule for this pulse information, it was possible to distinguish one's pulses. In this simulation, as the LIDAR sensor (a) transmitted 27 pulses to one target point, the many reflected waves were received by LIDAR sensor (b). LIDAR (b) was able to receive 28 reflected pulses from one target point. One of them was its own reflected pulse, and 27 pulses were from LIDAR sensor (a). These higher reflected pulses led to higher mutual interference, and then LIDAR sensor (b) had much more mutual interference than the second step. On the other hand, even when the reflected wave of the laser pulse emitted from LIDAR sensor (b) was received by LIDAR sensor (a), it did 
not decode the spreading code and was removed, so there was no mutual interference in LIDAR sensor (a).

\subsection{Spatial and Temporal Locality of Mutual Interference}

Table 9 and Figure 14a show the occurrence of interference in consecutive interfered angles from the result of LIDAR sensor (b) in the second and third steps. Mutual interferences mostly occurred in a single angle on the same scan line. In some cases, mutual interferences occurred in up to 20 consecutive angles. The results show that interference had temporal locality. If a particular angle interfered at a given time, then it was likely that nearby angles would interfere soon. Single mutual interference can be ignored as noise or error. It is hard to ignore consecutive mutual interference on the same line because the real object is possible.

Table 9. Occurrence of consecutive mutual interference in a line on the LIDAR sensor (b).

\begin{tabular}{|c|c|c|c|c|c|c|c|c|c|c|c|c|c|c|c|c|c|c|c|c|c|}
\hline Scene & Mode & 1 & 2 & 3 & 4 & 5 & 6 & 7 & 8 & 9 & 10 & 11 & 12 & 13 & 14 & 15 & 16 & 17 & 18 & 19 & 20 \\
\hline \multirow{2}{*}{ \# 1} & Single-pulse & 3582 & 2097 & 869 & 725 & 596 & 424 & 360 & 352 & 275 & 219 & 189 & 173 & 112 & 68 & 52 & 51 & 45 & 33 & 3 & \\
\hline & Coded pulses & $4,918,635$ & 90,564 & 15,602 & 11,425 & 5681 & 1762 & 889 & 808 & 483 & 322 & 284 & 240 & 210 & 189 & 178 & 156 & 112 & 74 & 70 & 45 \\
\hline \multirow{2}{*}{ \# 2} & & 21,901 & 2175 & 1129 & 560 & 437 & 163 & 102 & 70 & 67 & 61 & 41 & 10 & 6 & 5 & 0 & 1 & & 0 & 0 & \\
\hline & Coded pulses & 129,496 & 11,168 & 2181 & 755 & 625 & 615 & 562 & 289 & 263 & 225 & 199 & 134 & 90 & 79 & 41 & 1 & 1 & 0 & 0 & \\
\hline \multirow{2}{*}{ \# 3} & & 2088 & 1171 & 1107 & 518 & 473 & 409 & 369 & 314 & 293 & 261 & 207 & 132 & 102 & 67 & 59 & 59 & 33 & 22 & 0 & \\
\hline & Coded pulses & 357,578 & 43,521 & 13,620 & 5773 & 3056 & 2023 & 1120 & 922 & 453 & 395 & 393 & 256 & 171 & 170 & 109 & 74 & 58 & 16 & 0 & \\
\hline
\end{tabular}

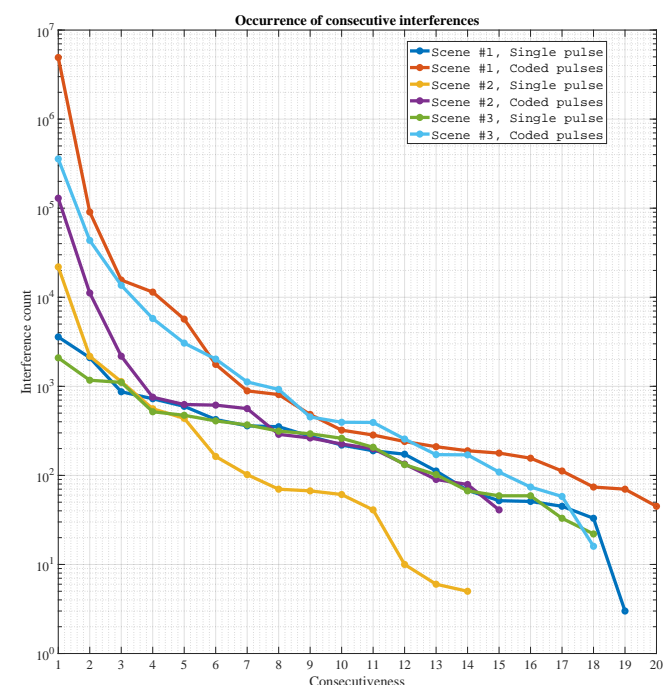

(a)

gure 14. Occurrence of consecutive mutual interference. (a) Consecutive mutual interference in a line; (b) consecutive mutual interference at the same angle.

Table 10 and Figure 14b show how long the event occurred in the same angles in the consecutive interfered lines from the result of sensor (b) in the second and third steps. Mutual interferences mostly occurred in single lines on the same scan angle. In some cases, mutual interferences occurred in up to 12 consecutive lines at the same scan angle. The results show that interference had spatial locality. If a particular line interfered at a given time, the same location would likely interfere shortly. Single mutual interference can be ignored as noise or error. It is hard to ignore consecutive mutual interference at the same angle because the real object is possible. 
Table 10. Occurrence of consecutive mutual interference at the same angle on the LIDAR sensor (b).

\begin{tabular}{ccrrrrrrrrrrrr}
\hline Scene & Mode & $\mathbf{1}$ & $\mathbf{2}$ & $\mathbf{3}$ & $\mathbf{4}$ & $\mathbf{5}$ & $\mathbf{6}$ & $\mathbf{7}$ & $\mathbf{8}$ & $\mathbf{9}$ & $\mathbf{1 0}$ & $\mathbf{1 1}$ & $\mathbf{1 2}$ \\
\hline \multirow{2}{*}{$\# 1$} & Single-pulse & 21,669 & 1628 & 221 & 214 & 101 & 77 & 56 & 0 & 0 & 0 & 0 & 0 \\
& Coded pulses & 2085 & 1448 & 346 & 267 & 155 & 138 & 44 & 0 & 0 & 0 & 0 & 0 \\
\hline \multirow{2}{*}{$\# 2$} & Single-pulse & 720 & 211 & 155 & 147 & 30 & 29 & 14 & 0 & 0 & 0 & 0 & 0 \\
& Coded pulses & 1283 & 617 & 532 & 198 & 149 & 129 & 84 & 83 & 73 & 65 & 12 & 6 \\
\hline \multirow{2}{*}{$\# 3$} & Single-pulse & 8793 & 1051 & 455 & 262 & 201 & 106 & 53 & 32 & 30 & 14 & 0 & 0 \\
& Coded pulses & 9992 & 564 & 491 & 393 & 118 & 51 & 35 & 18 & 8 & 0 & 0 & 0 \\
\hline
\end{tabular}

\subsection{Fourth Step: Elimination of Mutual Interference between Coded Pulse Stream Modes}

Figures 15-17 are the results of the fourth step in the simulation. They show the (2-D) distance grid map acquired by LIDAR sensor (a) and (b) in each scenario. As described in Section 4, two LIDAR sensors were operated simultaneously in the coded pulse stream mode. After simulation, we analyzed and determined the recorded data, as shown in Equation (6), and plotted correct distances as green color-filled square symbols and incorrect distances as red cross symbols.

In the fourth step, two LIDAR sensors operated in the same coded pulse stream mode, and they only transmitted and received pulse streams generated by their own specific rules and ignored any others. Even if multiple reflected pulse streams were received by sending multiple pulses to one target from another LIDAR sensor, any signals that did not conform to their rules were removed. Eventually, mutual interference did not occur. In addition, since the distance to a single target was calculated using multiple measurement results instead of one, the distance accuracy was significantly improved $[40,41]$.

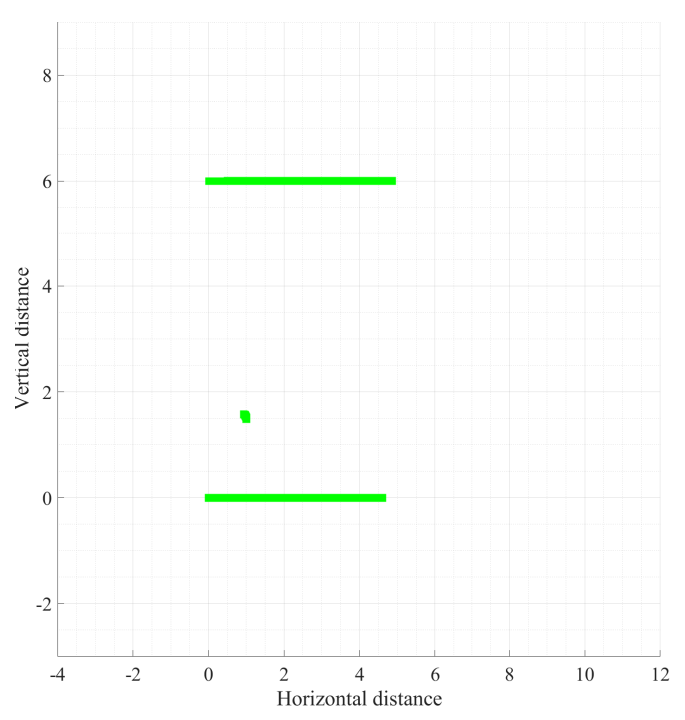

(a)

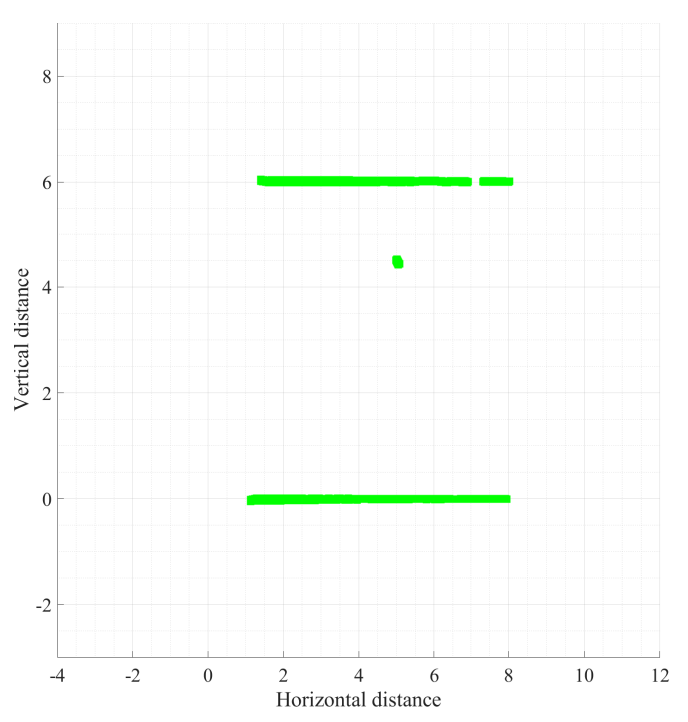

(b)

Figure 15. Two-dimensional distance grid maps of scene \#1 show correct distances and incorrect distances measured by each LIDAR sensor in the fourth step. (a) From LIDAR sensor (a)'s point of view; (b) LIDAR sensor (b)'s point of view. 


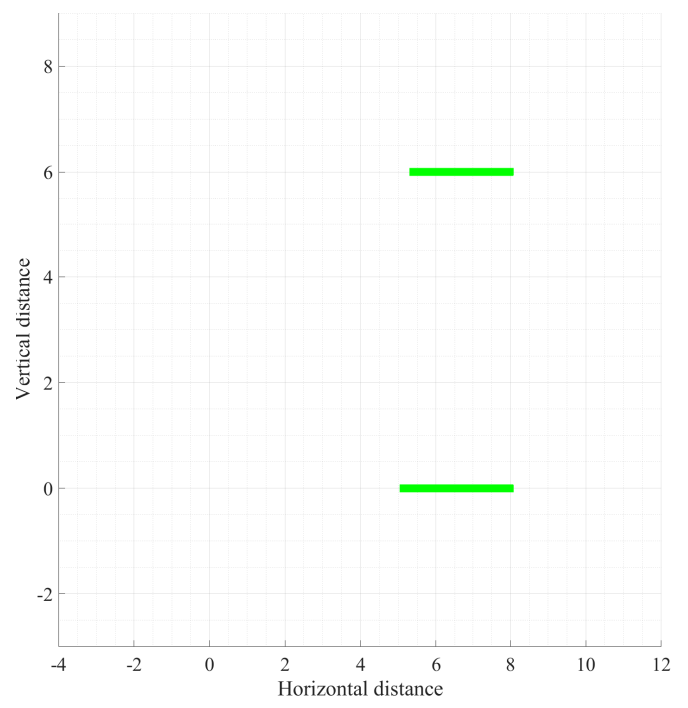

(a)

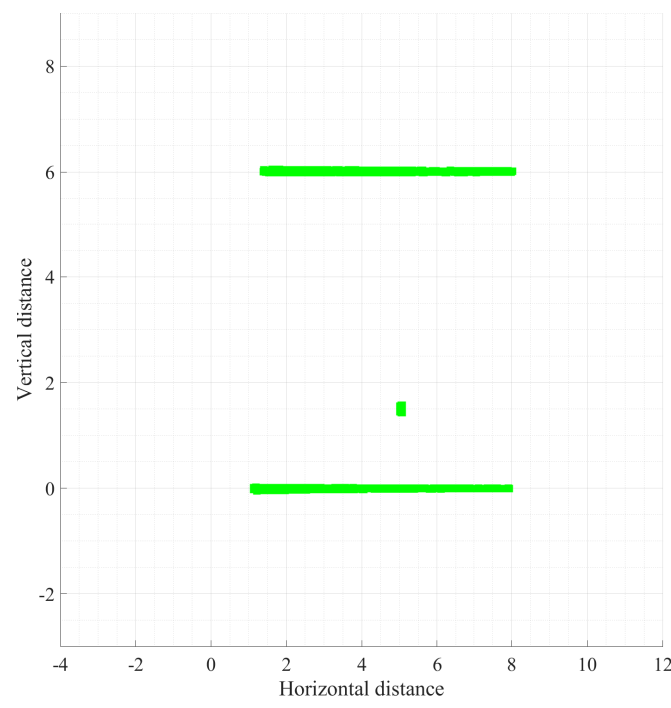

(b)

Figure 16. Two-dimensional distance grid maps of scene \#2 show correct distances and incorrect distances measured by each LIDAR sensor in the fourth step. (a) From LIDAR sensor @'s point of view; (b) LIDAR sensor (b)'s point of view.

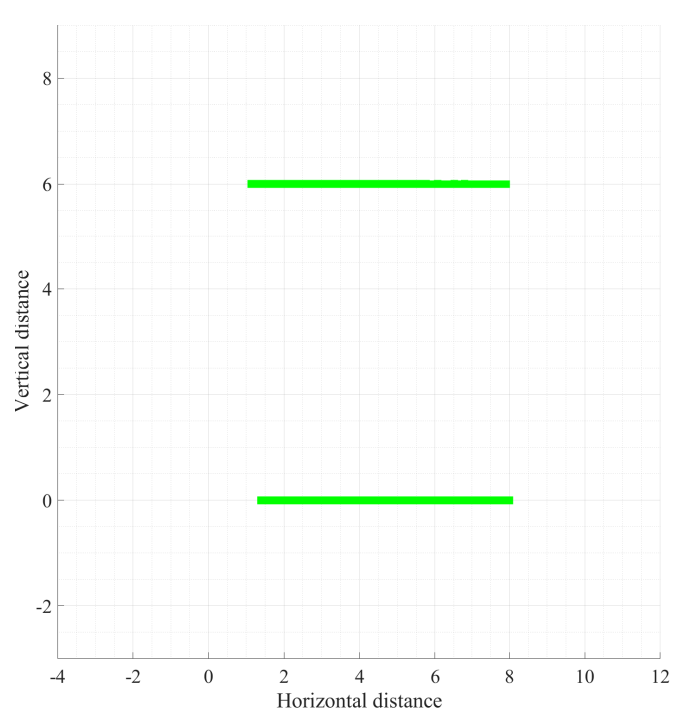

(a)

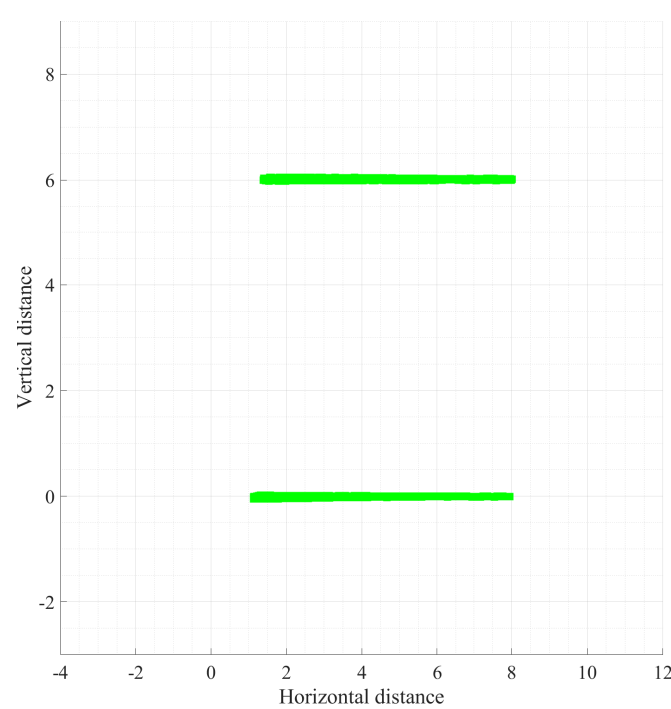

(b)

Figure 17. Two-dimensional distance grid maps of scene \#3 show correct distances and incorrect distances measured by each LIDAR sensor in the fourth step. (a) From LIDAR sensor @'s point of view; (b) LIDAR sensor (b)'s point of view.

\section{Conclusions}

Active sensors using a wireless domain, such as a radar or a LIDAR sensor, are not free from mutual interference. In the EU, through the MOSAIM project, a study was performed on how mutual interference occurs in vehicle radar sensors. It confirmed that direct and indirect mutual interference could occur in various environments where a vehicle can drive. Unlike a radar sensor, a LIDAR sensor has a very small divergence angle, and direct mutual interferences rarely occur. Since a LIDAR sensor creates a significant intensity of reflected waves compared to a radar sensor, indirect mutual interference is likely to occur. Several preceding studies on the occurrence and resolution of mutual interference in LIDAR sensors were limited to using a single plane.

We performed simulations in which a LIDAR sensor operated in three scenarios where indirect mutual interference occurred in a radar sensor and confirmed that mutual 
interference could occur in all situations. These mutual interferences have temporal and spatial locality that could lead to real objects. In this study, these simulations showed that mutual interference occurs in LIDAR sensors using multi-planes and can be effectively prevented by using a coded pulse stream. The coded pulse stream method proposed in this paper randomly generates necessary information and performs encoding and decoding processes based on this. It is shown that the alien signals that caused mutual interference were very effectively removed. It is necessary to study the coded pulse stream so that it can be used when there are many LiDAR sensors in a dense space in the future. Furthermore, it is necessary to study side effects that may occur when using coded pulse streams. Due to the significant differences between the simulations and the actual environment, the signal reflected by irregular objects can destroy the encoded pulse stream in reality. So, LIDAR sensors cannot decode the received encoded pulse stream correctly.

Author Contributions: Conceptualization, G.K. and J.E.; data curation, G.K.; formal analysis, G.K.; funding acquisition, Y.P.; investigation, J.E.; methodology, G.K.; project administration, Y.P.; resources, G.K.; software, G.K.; supervision, Y.P.; validation, G.K. and J.E.; visualization, G.K.; writing—original draft, G.K.; writing — review and editing, J.E. All authors read and agreed to the published version of the manuscript.

Funding: This work was supported in part by the Basic Science Research Program through the National Research Foundation of Korea (NRF) funded by the Ministry of Education under Grant NRF-2021R1A6A1A03039493 and NRF-2021R1A2B5B02086773.

Institutional Review Board Statement: Not applicable.

Informed Consent Statement: Not applicable.

Data Availability Statement: Not applicable.

Conflicts of Interest: The authors declare no conflict of interest. The funders had no role in the design of the study; in the collection, analyses, or interpretation of data; in the writing of the manuscript; or in the decision to publish the results.

\begin{tabular}{|c|c|}
\hline \multicolumn{2}{|c|}{ Abbreviations } \\
\hline \multicolumn{2}{|c|}{ The following abbreviations are used in this manuscript: } \\
\hline 2-D & Two-dimensional \\
\hline $3-\mathrm{D}$ & Three-dimensional \\
\hline CHPC & Carrier-hopping prime code \\
\hline CRC & Cyclic redundancy check \\
\hline $\mathrm{dToF}$ & Direct time-of-flight \\
\hline FoV & Field of view \\
\hline ID & Identification \\
\hline LIDAR & Light detection and ranging \\
\hline MOSARIM & More safety for all by radar interference mitigation \\
\hline PRF & Pulse repetition frequency \\
\hline radar & Radio detection and ranging \\
\hline SNR & Signal-to-noise ratio \\
\hline ToF & Time-of-flight \\
\hline
\end{tabular}

\section{References}

1. De Ponte Müller, F. Survey on ranging sensors and cooperative techniques for relative positioning of vehicles. Sensors 2017, 17, 271.

2. Shi, W.; Alawieh, M.B.; Li, X.; Yu, H. Algorithm and hardware implementation for visual perception system in autonomous vehicle: A survey. Integration 2017, 59, 148-156.

3. Boulay, P.; Debray, A. LiDAR for Automotive and Indurstrial Applications 2021; Report; Yole Development: Lyon-Villeurbanne, France, 2021.

4. Van Nam, D.; Gon-Woo, K. Solid-State LiDAR based-SLAM: A Concise Review and Application. In Proceedings of the 2021 IEEE International Conference on Big Data and Smart Computing (BigComp), Bangkok, Thailand, 17-20 January 2021; IEEE: Bangkok, Thailand, 2021; pp. 302-305. 
5. $\quad$ Li, N.; Ho, C.P.; Wang, I.T.; Pitchappa, P.; Fu, Y.H.; Zhu, Y.; Lee, L.Y.T. Spectral imaging and spectral LIDAR systems: Moving toward compact nanophotonics-based sensing. Nanophotonics 2021, 10, 1437-1467.

6. Bi, S.; Yuan, C.; Liu, C.; Cheng, J.; Wang, W.; Cai, Y. A Survey of Low-Cost 3D Laser Scanning Technology. Appl. Sci. 2021, 11, 3938.

7. Roriz, R.; Cabral, J.; Gomes, T. Automotive LiDAR Technology: A Survey. IEEE Trans. Intell. Transp. Syst. 2021, Early Access, 1-16.

8. Mitropoulos, L.K.; Prevedouros, P.D.; Yu, X.A.; Nathanail, E.G. A Fuzzy and a Monte Carlo simulation approach to assess sustainability and rank vehicles in urban environment. Transp. Res. Procedia 2017, 24, 296-303.

9. Martino, L.; Read, J.; Elvira, V.; Louzada, F. Cooperative parallel particle filters for online model selection and applications to urban mobility. Digit. Signal Process. 2017, 60, 172-185.

10. Bastos, D.; Monteiro, P.P.; Oliveira, A.S.; Drummond, M.V. An Overview of LiDAR Requirements and Techniques for Autonomous Driving. In Proceedings of the 2021 Telecoms Conference (ConfTELE), Leiria, Portugal, 11-12 February 2021; IEEE: Leiria, Portugal, 2021; pp. 1-6.

11. Brooker, G.M. Mutual Interference of Millimeter-Wave RADAR Systems. IEEE T. Electromagn. C. 2007, 49, 170-181.

12. Alland, S.; Stark, W.; Ali, M.; Hegde, M. Interference in automotive radar systems: Characteristics, mitigation techniques, and current and future research. IEEE Signal Process. Mag. 2019, 36, 45-59.

13. Aydogdu, C.; Keskin, M.F.; Carvajal, G.K.; Eriksson, O.; Hellsten, H.; Herbertsson, H.; Nilsson, E.; Rydstrom, M.; Vanas, K.; Wymeersch, H. Radar interference mitigation for automated driving: Exploring proactive strategies. IEEE Signal Process. Mag. 2020, 37, 72-84.

14. Kui, L.; Huang, S.; Feng, Z. Interference Analysis for mmWave Automotive Radar Considering Blockage Effect. Sensors 2021, $21,3962$.

15. Kalman, R.E. A new approach to linear filtering and prediction problems. J. Basic Eng. Mar. 1960, 82, 35-45.

16. Carvalho, C.M.; Johannes, M.S.; Lopes, H.F.; Polson, N.G. Particle learning and smoothing. Stat. Sci. 2010, 25, 88-106.

17. Zhao, F.; Jiang, H.; Liu, Z. Recent development of automotive LiDAR technology, industry and trends. In Proceedings of the Eleventh International Conference on Digital Image Processing (ICDIP 2019), Guangzhou, China, 10-13 May 2019; International Society for Optics and Photonics: Bellingham, WA, USA, 2019; Volume 11179, p. 111794A.

18. Fischer, C.; Ahrholdt, M.; Ossowska, A.; Kunert, M.; John, A.; Pietsch, R.; Bodereau, F.; Hildebrandt, J.; Blöcher, H.; Meinel, H. Use cases description list for simulation scenarios; Rreport; The MOSARIM Consortium: 2010.

19. Ahrholdt, M.; Bodereau, F.; Fischer, C.; Goppelt, M.; Pietsch, R.; John, A.; Ossowska, A.; Kunert, M. Use cases description list for simulation scenarios; Rreport; The MOSARIM Consortium: 2010.

20. Schipper, T. Multi-interference modeling and effects; Rreport; The MOSARIM Consortium: 2012.

21. Aydogdu, C.; Keskin, M.F.; Garcia, N.; Wymeersch, H.; Bliss, D.W. RadChat: Spectrum sharing for automotive radar interference mitigation. IEEE Trans. Intell. Transp. Syst. 2019, 22, 416-429.

22. Schipper, T. Simulation of effects and impact of environment, traffic participants and infrastructure; Rreport; The MOSARIM Consortium: 2012.

23. Goppelt, M.; Blöcher, H.L.; Menzel, W. Automotive RADAR-Investigation of Mutual Interference Mechanisms. Adv. Radio Sci. 2010, 8, 55-60.

24. Kirmani, A.; Benedetti, A.; Chou, P.A. SPUMIC: Simultaneous Phase Unwrapping and Multipath Interference Cancellation in Time-of-Flight Cameras using Spectral Methods. In Proceedings of the 2013 IEEE International Conference on Multimedia and Expo (ICME'13), San Jose, CA, USA, 15-19 July 2013; IEEE: San Jose, CA, USA, 2013; pp. 1-6.

25. Goppelt, M.; Blocher, H.L.; Menzel, W. Analytical Investigation of Mutual Interference between Automotive FMCW RADAR Sensors. In Proceedings of the 2011 IEEE Microwave Conference (GeMIC'11), Darmstadt, Germany, 14-16 March 2011; IEEE: Darmstadt, Germany, 2011; pp. 1-4.

26. McManamon, P.F. Review of LADAR: A Historic, Yet Emerging, Sensor Technology with Rich Phenomenology. Opt. Eng. 2012, $51,060901$.

27. Dorrington, A.A.; Godbaz, J.P.; Cree, M.J.; Payne, A.D.; Streeter, L.V. Separating True Range Measurements from Multi-Path and Scattering Interference in Commercial Range Cameras. In Proceedings of SPIE-The International Society for Optical Engineering; SPIE: Bellingham, WA, USA, 2011; p. 786404.

28. Falie, D.; Buzuloiu, V. Noise Characteristics of 3D Time-of-Flight Cameras. In Proceedings of the 2007 IEEE International Symposium on Signals, Circuits and Systems (ISSCS'07), Iasi, Romania, 13-14 July 2007; IEEE: Iasi, Romania, 2007; Volume 1, pp. $1-4$

29. Guðmundsson, S.Á.; Aanæs, H.; Larsen, R. Environmental Effects on Measurement Uncertainties of Time-of-Flight Cameras. In Proceedings of the 2007 IEEE International Symposium on Signals, Circuits and Systems (ISSCS'07), Iasi, Romania, 13-14 July 2007; IEEE: Iasi, Romania, 2007; Volume 1, pp. 1-4.

30. Kim, G.; Eom, J.; Park, S.; Park, Y. Occurrence and Characteristics of Mutual Interference between LIDAR Scanners. In Proceedings of SPIE-Photon Counting Applications 2015; International Society for Optics and Photonics: San Diego, CA, USA, 2015; p. 95040K. https://doi.org/10.1117/12.2178502.

31. Kim, G.; Eom, J.; Park, Y. Investigation on the Occurrence of Mutual Interference between Pulsed Terrestrial LIDAR Scanners. In Proceedings of the 2015 IEEE Intelligent Vehicles Symposium (IV), Seoul, Korea, 28 June 28-1 July 2015; IEEE: Seoul, Korea, 2015; pp. 437-442. 
32. Kim, G.; Eom, J.; Hur, S.; Park, Y. Analysis on the Characteristics of Mutual Interference between Pulsed Terrestrial LIDAR Scanners. In Proceedings of the 2015 IEEE International Geoscience and Remote Sensing Symposium (IGARSS), Milan, Italy, 26-31 July 2015; IEEE: Milan, Italy, 2015; pp. 2151-2154.

33. Eom, J.; Kim, G.; Hur, S.; Park, Y. Assessment of Mutual Interference Potential and Impact with Off-the-Shelf Mobile LIDAR In Advanced Photonics 2018 (BGPP, IPR, NP, NOMA, Sensors, Networks, SPPCom, SOF); Optical Society of America: California, CA, USA, 2018; p. JTu2A.66.

34. Martins, P.M.S.B. Interference Analysis in Time of Flight LiDARs. Master's Thesis, Universidade de Aveiro, Aveiro, Portugal, 2019.

35. Popko, G.B.; Bao, Y.; Gaylord, T.K.; Valenta, C.R. Beam path intersections between two coplanar lidar scanners. Opt. Eng. 2019, 58,033103

36. Popko, G.B.; Gaylord, T.K.; Valenta, C.R. Geometric approximation model of inter-lidar interference. Opt. Eng. 2020, 59, 033104.

37. Hwang, I.P.; Lee, C.H. Mutual interferences of a true-random LiDAR with other LiDAR signals. IEEE Access 2020, 8, $124123-124133$.

38. Kawata, H.; Kamimura, S.; Ohya, A.; Iijima, J.; Yuta, S. Advanced Functions of the Scanning Laser Range Sensor for Environment Recognition in Mobile Robots. In Proceedings of the 2006 IEEE International Conference on Multisensor Fusion and Integration for Intelligent Systems, Heidelberg, Germany, 3-6 September 2006; IEEE: Heidelberg, Germany, 2006; pp. 414-419.

39. Lo, F.W.; Yang, G.C.; Lin, W.Y.; Glesk, I.; Kwong, W.C. 2-D optical-CDMA modulation with hard-limiting for automotive time-of-flight LIDAR. IEEE Photon. J. 2021, 13, 7200111.

40. Kim, G.; Park, Y. Independent Biaxial Scanning Light Detection and Ranging System Based on Coded Laser Pulses without Idle Listening Time. Sensors 2018, 18, 2943.

41. Kim, G.; Ashraf, I.; Eom, J.; Park, Y. Concurrent Firing Light Detection and Ranging System for Autonomous Vehicles. Remote Sens. 2021, 13, 1767. https://doi.org/10.3390/rs13091767.

42. Ghillino, E.; Virgillito, E.; Mena, P.V.; Scarmozzino, R.; Stoffer, R.; Richards, D.; Ghiasi, A.; Ferrari, A.; Cantono, M.; Carena, A.; et al. The synopsys software environment to design and simulate photonic integrated circuits: A case study for $400 \mathrm{~g}$ transmission. In Proceedings of the 2018 20th International Conference on Transparent Optical Networks (ICTON), Bucharest, Romania, 1-5 July 2018; IEEE: Bucharest, Romania, 2018, pp. 1-4. 